\title{
EXACT RIEMANN SOLUTIONS TO COMPRESSIBLE EULER EQUATIONS IN DUCTS WITH DISCONTINUOUS CROSS-SECTION
}

\author{
EE HAN*, MAREN HANTKE ${ }^{\dagger}$, AND GERALD WARNECKE $\ddagger$
}

\begin{abstract}
We determine completely the exact Riemann solutions for the system of Euler equations in a duct with discontinuous varying cross-section. The crucial point in solving the Riemann problem for hyperbolic system is the construction of the wave curves. To address the difficulty in the construction due to the nonstrict hyperbolicity of the underlying system, we introduce the L-M and $\mathrm{R}-\mathrm{M}$ curves in the velocity-pressure phase plane. The behaviors of the $\mathrm{L}-\mathrm{M}$ and $\mathrm{R}-\mathrm{M}$ curves for six basic cases are fully analyzed. Furthermore, we observe that in certain cases the $\mathrm{L}-\mathrm{M}$ and $\mathrm{R}-\mathrm{M}$ curves contain the bifurcation which leads to the non-uniqueness of the Riemann solutions. Nevertheless, all possible Riemann solutions including classical as well as resonant solutions are solved in a uniform framework for any given initial data.
\end{abstract}

Key words. shock waves, rarefaction waves, velocity function, stationary waves, resonant waves, Mach number, nonuniqueness solutions

AMS subject classifications. 65M06, 76M12, 35L60

1. Introduction. We consider the Euler equations modeling compressible fluid flows through a duct of variable cross-section with the area $a(x)$. They are given in the perturbation form

$$
\frac{\partial \mathbf{U}}{\partial t}+\frac{\partial \mathbf{F}(\mathbf{U})}{\partial x}=-\frac{a^{\prime}(x)}{a(x)} \mathbf{H}(\mathbf{U})
$$

where

$$
\mathbf{U}=\left[\begin{array}{c}
\rho \\
\rho v \\
\rho E
\end{array}\right], \quad \mathbf{F}(\mathbf{U})=\left[\begin{array}{c}
\rho v \\
\rho v^{2}+p \\
v(\rho E+p)
\end{array}\right], \quad \mathbf{H}(\mathbf{U})=\left[\begin{array}{c}
\rho v \\
\rho v^{2} \\
v(\rho E+p)
\end{array}\right]
$$

and the dependent variables $\rho, v$ and $p$ denote, respectively, the density, velocity and pressure of the fluid. The specific total energy is given as $E=e+\frac{v^{2}}{2}$, where $e$ is the internal energy. The polytropic equation of state

$$
e=\frac{p}{\rho(\gamma-1)}
$$

is used to close the system, where $\gamma$ is the ratio of specific heats and satisfies $1<\gamma<3$. There is a considerable literature devoted to the investigation of the Euler equations in a duct with variable cross section, for example Liu [12, 13], as well as Andrianov and Warnecke [1]. For more related works, see [2, 3, 6, 9, 14, 16, 11, 18], and the references cited therein.

A particular feature of the system (1.1) is the presence of the cross section area $a(x)$. This geometric variable is independent of the time and leads to a stationary

\footnotetext{
*Department of Mathematics, Magdeburg University, Universitaetsplatz 239106 Magdeburg, Germany (han.ee@st.ovgu.de).,

$\dagger$ Department of Mathematics, Magdeburg University, Universitaetsplatz 239106 Magdeburg, Germany (maren.hantke@ovgu.de).

$\ddagger$ Department of Mathematics, Magdeburg University, Universitaetsplatz 239106 Magdeburg, Germany (gerald.warnecke@ovgu.de).
} 
source. For the uniform duct area, i.e. $a^{\prime}(x)=0$, the system (1.1) becomes the usual homogeneous system of Euler equations

$$
\mathbf{U}_{t}+\mathbf{F}(\mathbf{U})_{x}=0 .
$$

This system has been extensively studied, see Evans [7] and Smoller [17]. It is well known that the solution to the Riemann problem for (1.4) consists of shock waves, rarefaction waves and contact discontinuities. These three elementary waves are selfsimilar and uniquely determined by the initial data.

For the perturbed system (1.1) the waves consist of the aforementioned elementary waves and a stationary wave which is determined by

$$
\mathbf{F}(\mathbf{U})_{x}=-\frac{a^{\prime}(x)}{a(x)} \mathbf{H}(\mathbf{U}) .
$$

Compared with the homogeneous Euler equations (1.4) the waves associated with the system (1.1) propagate in a much more complicated way as a result of variations of $a(x)$. Liu in $[12,13]$ studied flows in continuous, piecewise smooth ducts. Our results can be considered as limiting cases to [12]. We also obtain uniqueness for the expanding duct and non-uniqueness for the contracting duct. In [13] Liu introduced the important physical phenomenon of resonance, namely that waves of different families are not well separated and coincide. Particularly he revealed that the nonlinear resonance effects can cause instability of a flow field and change wave types in the solution. We address this phenomenon for the discontinuous duct flows.

Isaacson and Temple [10] complemented the system (1.1) with an additional trivial equation $a_{t}=0$. Andrianov and Warnecke [1], Goatin and LeFloch [9], LeFloch and Thanh [11], Thanh [18] followed the idea and studied the solutions to the corresponding Riemann problem. This additional equation $a_{t}=0$ introduces a linear degenerate field with a 0 speed eigenvalue. The strictly hyperbolic system (1.1) with a given function $a(x)$ is transformed into a system that is not hyperbolic everywhere in the state space. Due to the coincidence of eigenvectors the system becomes degenerate at sonic states, see Goatin and LeFloch [9]. In some sense the study of the extended system clarifies the problem of resonance. Another well known problem for the current nonconservative hyperbolic system is the occurrence of nonuniqueness, i.e. more than one solution is produced by given initial data. A typical example of a nonunique solution was observed by Andrianov and Warnecke in [1]. Moreover we note that this nonuniqueness problem also appears in Liu's results $[12,13]$. Nevertheless, the Riemann solution to the extended system remains the same as that to the system (1.1). Furthermore the extended system (1.1) with the trivial equation $a_{t}=0$ closely reflects properties of certain systems for two phase flows. From a mathematical point view it can be regarded as a submodel of the Baer-Nunziato model, see Andrianov and Warnecke [2].

Therefore, we adopt the form of the governing system as follows

$$
\left\{\begin{array}{l}
a_{t}=0, \\
\mathbf{U}_{t}+\mathbf{F}(\mathbf{U})_{x}=-\frac{a^{\prime}(x)}{a(x)} \mathbf{H}(\mathbf{U}) .
\end{array}\right.
$$

The purpose of this paper is to completely construct the exact Riemann solutions to the system (1.6) for arbitrary initial data. Previously, Marchesin and Paes-Leme [15] presented the Riemann solution for the reduced isothermal gas dynamics in a duct with a discontinuous cross-section. Andrianov and Warnecke [1] studied the exact 
Riemann solutions to (1.6) without resonance in an 'inverse' way. They considered certain wave types and intermediate states for which they determined the Riemann initial data. Their solutions are useful for testing numerical schemes. Clearly, the procedure is difficult to be generalized. Goatin and LeFloch [9] proved the existence of the Riemann solutions for the general inhomogeneous systems around the resonant states $\left(\mathbf{U}^{*}, a^{*}\right)$ under the assumption that the $k$-field is genuinely nonlinear and $\mathbf{l}_{k} \cdot \frac{\mathbf{H}(\mathbf{U})}{a(x)}\left(\mathbf{U}^{*}, a^{*}\right) \neq 0$, where $\mathbf{l}_{k}$ are the corresponding left $k$-eigenvectors. Recently, Thanh [18] constructed the Riemann solutions to (1.6) for large data. However, he did not present a comprehensive classification of all the possible cases and missed some solutions, such as e.g. the Riemann initial data in Table 4.6 satisfies the condition of Construction $N 1$ but involves the wave configuration $E$ not $F$, see Figures 4.17 and 4.18. However he claimed uniqueness for the Riemann problem whereas we show that cases of the nonouniqueness exists among the solutions. The objective of this paper is to address these open points.

Note that the Riemann problem solutions for strictly hyperbolic systems are given by the connection of various wave curves $T_{k}$ in the state space. The curve $T_{k}$ is associated with the $k$-characteristic field of the system and defined below, see also Evans [7]. Hence the crucial point in solving the Riemann problem for hyperbolic systems is the construction as well as the connection of all wave curves. There are four wave curves for our system (1.6). The first and third wave curves are given by the physically relevant parts of the rarefaction and shock curves. The first, second and third wave curves are inherited from the system (1.4). Moreover an additional stationary wave curve is defined by the ODE system (1.5). However the major problem is how to connect these components. We know that the mutual positions of the stationary wave curve with respect to the rest of the three elementary waves can't be determined a priori.

Marchesin and Paes-Leme [15] solved the Riemann problem for isothermal system by attaching the stationary wave curve to the first and third wave curves. We extend their ideas to the governing system by introducing the $\mathrm{L}-\mathrm{M}$ and $\mathrm{R}-\mathrm{M}$ curves. We take into account the stationary wave curves by deriving the velocity function. Owing to this function the $\mathrm{L}-\mathrm{M}$ and $\mathrm{R}-\mathrm{M}$ curves can be related to the initial Mach number and variations of the duct area. There are six different types of basic cases for each curve. We carefully study the monotonic and continuous properties of the $\mathrm{L}-\mathrm{M}$ and $\mathrm{R}-\mathrm{M}$ curves in each case. Observe that a bifurcation occurs for certain cases. This introduces nonunique solutions. In brief we completely solve the Euler equation in a duct with discontinuous diameters for any Riemann initial data. The resulting solutions can be extended to the compressible fluid flows permeating into a porous bed [14], the shallow water equation with topography [8], and two phase flows of Baer-Nunziato model [2].

The organization of the paper. We briefly review the fundamental concepts and notions for the governing system in Section 2. In Section 3 we discuss the stationary wave curves in terms of solving the outflow state in the converging and expanding monotone ducts. Our main focus is in Section 4, which contains the definition of the $\mathrm{L}-\mathrm{M}$ and $\mathrm{R}-\mathrm{M}$ curves, also the algorithm for the exact solutions. All the possible wave configurations with positive intermediate velocity are illustrated in this section. Finally we conclude this paper in Section 5. 
2. Preliminaries. We now derive the quasi linear form of the system (1.6). Set $\mathbf{V}=(a, \rho, v, p)^{T}$, then

$$
\mathbf{V}_{t}+\mathbf{A}(\mathbf{V}) \mathbf{V}_{x}=0
$$

where the Jacobian matrix $\mathbf{A}(\mathbf{V})$ is in the form

$$
\mathbf{A}(\mathbf{V})=\left(\begin{array}{cccc}
0 & 0 & 0 & 0 \\
\frac{\rho v}{a} & v & \rho & 0 \\
0 & 0 & v & \frac{1}{\rho} \\
\frac{\gamma p}{a} & 0 & \gamma p & v
\end{array}\right)
$$

Observe that the matrix $\mathbf{A}(\mathbf{V})$ has four eigenvalues

$$
\lambda_{0}=0, \quad \lambda_{1}=v-c, \quad \lambda_{2}=v, \quad \lambda_{3}=v+c .
$$

They are referred to as the characteristic speeds. We emphasize that the system (1.6) is not strictly hyperbolic as a result of the fact that $\lambda_{0}$ can coincide with any of three other eigenvalues. The corresponding right eigenvectors are

$$
\mathbf{R}_{0}=\left(\begin{array}{c}
\frac{a\left(c^{2}-v^{2}\right)}{\rho v^{2}} \\
1 \\
-\frac{c^{2}}{\rho v} \\
c^{2}
\end{array}\right), \quad \mathbf{R}_{1}=\left(\begin{array}{c}
0 \\
1 \\
-\frac{c}{\rho} \\
c^{2}
\end{array}\right), \quad \mathbf{R}_{2}=\left(\begin{array}{c}
0 \\
1 \\
0 \\
0
\end{array}\right), \quad \mathbf{R}_{3}=\left(\begin{array}{c}
0 \\
1 \\
\frac{c}{\rho} \\
c^{2}
\end{array}\right)
$$

Direct calculation yields that

$$
\mathbf{R}_{0} \rightarrow \mathbf{R}_{k} \quad \text { as } \quad \lambda_{k} \rightarrow 0 \quad \text { when } \quad k=1,3 .
$$

Consequently the system (1.6) is degenerate on the states at which the eigenvalues $\lambda_{1}$ or $\lambda_{3}$ coincide with $\lambda_{0}$. Of course this violates the definition of hyperbolicity.

The initial data for Riemann problem of the system (1.6) are

$$
(a, \mathbf{U})(x, 0)= \begin{cases}\left(a_{L}, \mathbf{U}_{L}\right), & x<0 \\ \left(a_{R}, \mathbf{U}_{R}\right), & x>0\end{cases}
$$

where $a_{q}, \mathbf{U}_{q}$ with $q=L$ or $R$ are constant. We use $k$-waves, $k=0,1,2,3$, to denote waves associated to the $k$-characteristic fields when the eigenvalues are distinct from each other. Here the $2-$ waves are the contact discontinuities. In addition the $1-$ and 3 -waves are shocks or rarefaction. The 0 -wave is a standing wave defined by the ODE system (1.5). We observe that the perturbation system (1.6) can be reformulated in a conservative form for the given area $a(x)$

$$
\begin{aligned}
& a_{t}=0, \\
& (a \rho)_{t}+(a \rho v)_{x}=0, \\
& (a \rho v)_{t}+\left(a \rho v^{2}+p\right)_{x}=p a_{x}, \\
& (a \rho E)_{t}+(a \rho(E+p))_{x}=0 .
\end{aligned}
$$

Note that for the Riemann data (2.5) the term $p a_{x}$ plays a role only at $x=0$ where the stationary wave is located. Therefore everywhere away from the stationary wave the system (1.6) reduces to the usual Euler equations.

Details on these elementary waves can be found in any mathematical book on gas dynamics. The formulas in this paper particularly refer to Toro [19]. We now review them in short. 
2.1. Shock waves. Shock waves are discontinuous waves associated with the 1or 3-characteristic field. For a given state $\mathbf{U}_{q}$, the admissible shock curve $S_{k}\left(\mathbf{U}_{q}\right)$, $k=1,2$, as the set of states satisfies

$$
S_{k}\left(\mathbf{U}_{q}\right)=\left\{\mathbf{U} \mid \mathbf{F}(\mathbf{U})-\mathbf{F}\left(\mathbf{U}_{q}\right)=\sigma_{k}\left(\mathbf{U}-\mathbf{U}_{q}\right) \text { with } p>p_{q}\right\},
$$

where $\sigma_{k}$ is the shock speed given as

$$
\sigma_{k}=v_{q} \pm c_{q} \sqrt{\left(\frac{\gamma+1}{2 \gamma}\right)\left(\frac{p}{p_{q}}\right)+\left(\frac{\gamma-1}{2 \gamma}\right)} .
$$

Physically for any state $\mathbf{U} \in S_{k}\left(\mathbf{U}_{q}\right)$ we have

$$
\left\{\begin{array}{l}
v=v_{q} \pm\left(p-p_{q}\right)\left[\frac{\frac{2}{(\gamma+1) \rho_{q}}}{p+\frac{\gamma-1}{\gamma+1} p_{q}}\right]^{\frac{1}{2}} \\
\rho=\rho_{q}\left[\frac{\frac{\gamma-1}{\gamma+1}+\frac{p}{p_{q}}}{\left(\frac{\gamma-1}{\gamma+1}\right)\left(\frac{p}{p_{q}}\right)+1}\right]
\end{array}\right.
$$

where the " - " operator is taken when $k=1$ and the " + " operator is taken when $k=3$. We consider three components of the curve $S_{k}\left(\mathbf{U}_{q}\right)$, namely,

$$
\begin{aligned}
S_{k}^{ \pm}\left(\mathbf{U}_{q}\right) & =\left\{\mathbf{U} \mid \mathbf{U} \in S_{k}\left(\mathbf{U}_{q}\right) \text { and } \sigma_{k}\left(\mathbf{U}_{q}, \mathbf{U}\right) \gtrless 0\right\}, \\
S_{k}^{0}\left(\mathbf{U}_{q}\right) & =\left\{\mathbf{U} \mid \mathbf{U} \in S_{k}\left(\mathbf{U}_{q}\right) \text { and } \sigma_{k}\left(\mathbf{U}_{q}, \mathbf{U}\right)=0\right\} .
\end{aligned}
$$

2.2. Rarefaction waves. Rarefaction waves are continuous waves associated with the 1-and 3-characteristic fields. It is known that the rarefaction curves $R_{k}\left(\mathbf{U}_{q}\right)$, $k=1,3$, are the integral curves of the vector field $\mathbf{R}_{k} \cdot \nabla$ through $\mathbf{U}_{q}$. Any state $\mathbf{U}$ on the admissible curves $R_{k}\left(\mathbf{U}_{q}\right)$ satisfies the conditions of Riemann invariants

$$
R_{k}\left(\mathbf{U}_{q}\right)=\left\{\mathbf{U} \mid v \pm \frac{2 c}{\gamma-1}=v_{q} \pm \frac{2 c_{q}}{\gamma-1} ; \frac{p}{\rho^{\gamma}}=\frac{p_{q}}{\rho_{q}^{\gamma}} \text { with } p \leq p_{q}\right\}
$$

where the "+ " operator is taken when $k=1$ and the " - " operator is taken when $k=3$. The rarefaction wave has a fan-type shape and is enclosed by two bounding characteristics corresponding to the head and the tail of the waves, for example, when $k=1$ the head is $v_{L}-c_{L}$ and the tail is $v_{M}-c_{M}^{L}$; when $k=3$, the head is $v_{R}+c_{R}$ and the tail is $v_{M}+c_{M}^{R}$, where $v_{M}$ is the velocity of the intermediate state while $c_{M}^{L}$ and $c_{M}^{R}$ are, respectively, the corresponding left and right sound speed. The details can be found in the subsequent sections.

2.3. Contact discontinuity. The contact discontinuities are associated with the 2 -characteristic field. The velocity and pressure remains continuous but the density may jump across it. We use $\rho_{m}^{L}$ and $\rho_{m}^{R}$ to denote the left and right densities. From the relationship across the rarefaction or shock waves, we have $\rho_{m}^{q}=g_{q}\left(p ; \mathbf{U}_{q}\right)$, where

$$
g_{q}\left(p ; \mathbf{U}_{q}\right)= \begin{cases}\rho_{q}\left[\frac{\frac{\gamma-1}{\gamma+1}+\left(\frac{p}{p_{q}}\right)}{\frac{\gamma-1}{\gamma+1}\left(\frac{p}{p_{q}}\right)+1}\right], & \text { if } p>p_{q}, \\ \rho_{q}\left(\frac{p}{p_{q}}\right)^{\frac{1}{\gamma}}, & \text { if } p \leq p_{q} .\end{cases}
$$

The details refer to Toro [19]. 
2.4. Riemann solutions to the Euler equations. As is generally known there is a one-to-one correlation between Riemann solutions considered in the space-time $(x, t)$ plane and a set of parameterized, one dimensional wave curves in phase space. Hence solving the Riemann problem in the $(x, t)$ plane can be transformed into the construction of wave curves in the phase plane. The basic idea of the construction is to connect the wave curves associated to each $k$-characteristic field according to the mutual position of the eigenvalues. To be more precisely we use the usual Euler equation (1.4) as an example. The Riemann initial data for the system (1.4) are

$$
\mathbf{U}(x, 0)= \begin{cases}\mathbf{U}_{L}, & x<0, \\ \mathbf{U}_{R}, & x>0 .\end{cases}
$$

Note that the solution to the system (1.4), (2.13) is self-similar and consists of a 1-wave, a 2-wave which is the contact discontinuity, and a 3 -wave. Here the 1and 3 -waves are the shock or rarefaction waves. Since the velocity and pressure are continuous across the contact discontinuity, we project all states into the $(v, p)$ phase plane and define $1-\mathrm{M}$ curve $T_{1}\left(\mathbf{U}_{L}\right)$ and $3-\mathrm{M}$ curve $T_{3}\left(\mathbf{U}_{R}\right)$, which are defined in the following

$$
T_{k}\left(\mathbf{U}_{q}\right)=S_{k}\left(\mathbf{U}_{q}\right) \cup R_{k}\left(\mathbf{U}_{q}\right),
$$

where $\mathbf{U}_{q}$ are the initial states and $q$ is $L$ or $R$.

The 1-M curve $T_{1}\left(\mathbf{U}_{L}\right)$ is the set of states which can be connected to the left state $\mathbf{U}_{L}$ by the 1 -wave. Similarly the $3-\mathrm{M}$ curve $T_{3}\left(\mathbf{U}_{R}\right)$ is set of states which can be connected to the right states $\mathbf{U}_{R}$ by the 3 -wave. We observe that $T_{1}\left(\mathbf{U}_{L}\right)$ is a decreasing curve and $T_{3}\left(\mathbf{U}_{R}\right)$ is an increasing curve in the $(v, p)$ phase plane. The wave curves $T_{k}\left(\mathbf{U}_{q}\right), k=1,3$ are $C^{\infty}$ curves everywhere except at $\mathbf{U}_{q}$, where they are $C^{2}$, see Courant and Friedrichs [4]. In this paper we always assume that two curves $T_{1}\left(\mathbf{U}_{L}\right)$ and $T_{3}\left(\mathbf{U}_{R}\right)$ intersect at the state $\left(v_{M}, p_{M}\right)$, i.e. the appearance of a vacuum state in the solutions is excluded. To ensure this point the Riemann initial data are restricted by the constraint

$$
\frac{2}{\gamma-1}\left(c_{L}+c_{R}\right)-\left(v_{R}-v_{L}\right)>0
$$

Therefore, the wave curves of the exact Riemann solution in the $(v, p)$ phase space is the segment of $1-\mathrm{M}$ curve $T_{1}\left(\mathbf{U}_{L}\right)$ starting from the state $\mathbf{U}_{L}$ ending at the state $\mathbf{U}_{M}$, combining the segment of $3-\mathrm{M}$ curve $T_{3}\left(\mathbf{U}_{R}\right)$ from the state $\left(v_{M}, p_{M}\right)$ to the state $\mathbf{U}_{R}$. Thus the corresponding Riemann solution in the $(x, t)$ plane consists of the left state $\mathbf{U}_{L}$, a 1-wave (shock or rarefaction), the left intermediate state $\left(\rho_{m}^{L}, v_{m}, p_{m}\right)$, a contact discontinuity, the right intermediate state $\left(\rho_{m}^{R}, v_{m}, p_{m}\right)$, a 3-wave (shock or rarefaction), and the right state $\mathbf{U}_{R}$, where $\rho_{m}^{q}=g_{q}\left(p_{m} ; \mathbf{U}_{q}\right)$ with $q=L$ and $R$.

Return to our governing problem (1.6) with Riemann initial data (2.5). Obviously due to the fact that the location of the $k$-characteristic fields, $k=1,2,3$, and 0 characteristic field can not be estimated a priori, the components of the wave curves are difficult to be determined. To deal with this problem, we study the stationary wave curve in the next section.

3. Stationary waves. For simplicity we assume that the flow is from left to right, i.e. a positive flow velocity. The relations for the stationary waves obtained by integrating the system (1.5) are

$$
a \rho v=\text { constant },
$$




$$
\begin{aligned}
\frac{p}{\rho^{\gamma}} & =\text { constant }, \\
\frac{v^{2}}{2}+\frac{c^{2}}{\gamma-1} & =\text { constant } .
\end{aligned}
$$

We derive the following relations motivated by Courant and Friedrichs [4, (145.05), (145.08)].

$$
\begin{aligned}
\frac{1}{a} \frac{d a}{d x}+\frac{1}{\rho} \frac{d \rho}{d x}+\frac{1}{v} \frac{d v}{d x} & =0 \\
\frac{1}{\rho} \frac{d \rho}{d x}-\frac{2}{\gamma-1} \frac{1}{c} \frac{d c}{d x} & =0 \\
v \frac{d v}{d x}+\frac{2 c}{\gamma-1} \frac{d c}{d x} & =0
\end{aligned}
$$

Hence we obtain

$$
\frac{1}{a} \frac{d a}{d x}=\left(\frac{v^{2}}{c^{2}}-1\right) \frac{1}{v} \frac{d v}{d x} .
$$

The relation (3.5) shows that for smooth flows in an expanding duct, i.e. $\frac{d a}{d x}>0$, the velocity increases when $v>c$ and decreases when $0<v<c$. Of course the opposite relations hold for a contracting duct $\frac{d a}{d x}<0$ with respect to the positive velocity. An important consequence can also be inferred from the relation (3.5), that the quantity $a$ as a function of $x$ has a minimum at the sonic state $v^{2}=c^{2}$. Therefore, to avoid the appearance of the sonic state inside the duct, we consider ducts with the strictly monotone geometry, i.e. $\frac{d a}{d x}>0$ or $\frac{d a}{d x}<0$.

Proposition 3.1. For a stationary wave with positive velocity in a strictly monotonic duct with $a_{-}$as the inflow area and $a_{+}$as the outflow area, we have

1. If $a_{-}>a_{+}$, the sonic state can only appear as the outflow state.

2. If $a_{-}<a_{+}$, the sonic state can only appear as the inflow state.

3.1. The stationary flow in a strictly monotone duct. For simplicity we use $\mathbf{U}=\mathbf{J}\left(a_{+} ; \mathbf{U}_{-}, a_{-}\right)$to denote the state that can be connected to $\mathbf{U}_{-}$by a stationary flow in a monotonic duct with the area varying from $a_{-}$to $a_{+}$. Hereafter the values with " - " subscript are the inflow variables. Due to the nonlinear relations (3.1) the two states $\mathbf{U}_{-}$and $\mathbf{U}$ satisfy

$$
\begin{aligned}
a_{-} \rho_{-} v_{-} & =a_{+} \rho v, \\
\frac{p_{-}}{\rho_{-}^{\gamma}} & =\frac{p}{\rho^{\gamma}}, \\
\frac{v_{-}^{2}}{2}+\frac{c_{-}^{2}}{\gamma-1} & =\frac{v^{2}}{2}+\frac{c^{2}}{\gamma-1} .
\end{aligned}
$$

The formula (3.6) implies that the following conditions hold

1. $v_{-}$and $v$ have the same sign,

2. $v_{-}=0 \Longleftrightarrow v=0$.

Definitely the stationary flow vanishes when $v_{-}=0$, thus in the sequel we assume that $v_{-}>0$ unless stated explicitly.

Our aim is to obtain the outflow state $\mathbf{U}$ for given ducts with the inflow area $a_{-}$and the outflow area $a_{+}$, as well as the inflow state $\mathbf{U}_{-}$. Since the sound speed 
$c^{2}=\frac{\gamma p}{\rho}$, due to the isotropic relation (3.7) we have

$$
c^{2}=c_{-}^{2}\left(\frac{\rho}{\rho_{-}}\right)^{\gamma-1}
$$

Now taking (3.6) into (3.9), we obtain a relation for sound speeds

$$
c^{2}=c_{-}^{2}\left(\frac{a_{-} v_{-}}{a v}\right)^{\gamma-1}
$$

Consequently, a velocity function $\Psi\left(v ; \mathbf{V}_{-}, a_{+}\right)$is derived from the relation (3.8) by inserting (3.10) into it

$$
\Psi\left(v ; \mathbf{V}_{-}, a_{+}\right):=\frac{v^{2}}{2}+\frac{c_{-}^{2}}{\gamma-1}\left(\frac{a_{-} v_{-}}{a_{+} v}\right)^{\gamma-1}-\frac{v_{-}^{2}}{2}-\frac{c_{-}^{2}}{\gamma-1}
$$

where $v$ is the velocity and $\mathbf{V}_{-}=\left(a_{-}, \rho_{-}, v_{-}, p_{-}\right)^{T}$. Here $\mathbf{V}_{-}$, and $a_{+}$are taken as parameters. The solutions to the equation $\Psi\left(v ; \mathbf{V}_{-}, a_{+}\right)=0$ are the velocity of the corresponding outflow state. To calculate them the behavior of the velocity function is analyzed in the following lemma.

LEMmA 3.2. Consider $v^{*}=v_{-}\left(\frac{a_{-}}{a_{+}}\right)^{\frac{\gamma-1}{\gamma+1}}\left(\frac{c_{-}}{v_{-}}\right)^{\frac{2}{\gamma+1}}$, then the velocity function $\Psi\left(v ; \mathbf{V}_{-}, a_{+}\right)$has the following properties

1. $\Psi\left(v ; \mathbf{V}_{-}, a_{+}\right)$decreases if $v<v^{*}$;

2. $\Psi\left(v ; \mathbf{V}_{-}, a_{+}\right)$increases if $v>v^{*}$;

3. $\Psi\left(v^{*} ; \mathbf{V}_{-}, a_{+}\right)$has the minimum value at $v=v^{*}$ and there $v^{*}=c^{*}$, where $c^{*}$ is the corresponding sound speed.

Proof. The velocity function $\Psi\left(v ; \mathbf{V}_{-}, a_{+}\right)$is smooth since if $v_{-}>0$ the existence region for $v$ is $v>0$ also if $v_{-}<0$ then $v<0$. Therefore the derivative of $\Psi\left(v ; \mathbf{V}_{-}, a_{+}\right)$ is

$$
\frac{\partial \Psi\left(v ; \mathbf{V}_{-}, a_{+}\right)}{\partial v}=\frac{v^{\gamma+1}-\frac{a_{-}}{a_{+}} c_{-}^{2} v_{-}^{\gamma-1}}{v^{\gamma}} .
$$

Consequently we get

$$
\frac{\partial \Psi\left(v ; \mathbf{V}_{-}, a_{+}\right)}{\partial v} \begin{cases}<0, & \text { if } v<v^{*} \\ =0, & \text { if } v=v^{*} \\ >0, & \text { if } v>v^{*}\end{cases}
$$

It follows that the velocity function $\Psi\left(v ; \mathbf{V}_{-}, a_{+}\right)$is decreasing when $v<v^{*}$ and increasing when $v>v^{*}$ and has the minimum value at $v=v^{*}$.

Taking the relationship (3.10) into (3.12), we get the formula

$$
v \frac{\partial \Psi}{\partial v}\left(v ; \mathbf{V}_{-}, a_{+}\right)=v^{2}-c^{2}
$$

Since $\frac{\partial \Psi\left(v^{*} ; \mathbf{V}_{-}, a_{+}\right)}{\partial v}=0$ we obtain $v^{*}=c^{*}$. प

COROLlary 3.3. Lemma 3.2 shows that the equation $\Psi\left(v ; \mathbf{V}_{-}, a_{+}\right)=0$ may have two, one or no solutions. Further discussions are as follows,

1). If the minimum value $\Psi\left(v^{*} ; \mathbf{V}_{-}, a_{+}\right)<0$, the equation $\Psi\left(v ; \mathbf{V}_{-}, a_{+}\right)=0$ has two roots. Assume that the root closer to 0 is $v_{l}$ and the other one is $v_{r}, c_{l}$ and $c_{r}$ 
are the corresponding sound speeds. Then according to (3.14), $v_{l}^{2}-c_{l}^{2}<0$ and $v_{r}^{2}-c_{r}^{2}>0$. Because no flows can reach the sonic states inside a monotone duct, we can only take the one which satisfies

$$
\operatorname{sign}\left(v_{q}^{2}-c_{q}^{2}\right)=\operatorname{sign}\left(v_{-}^{2}-c_{-}^{2}\right)
$$

where $q=l$ or $r$. However if the inflow state $\mathbf{V}_{-}$is a sonic state, i.e. $v_{-}^{2}=c_{-}^{2}$, then (3.15) no longer holds. There are two possible solutions $v_{l}$ and $v_{r}$, which one is to be chosen depends on the state of the outflow for the specifical problem. We will consider this in Section 4.1.2.

2). If $\Psi\left(v^{*} ; \mathbf{V}_{-}, a_{+}\right)=0$, the equation $\Psi\left(v ; \mathbf{V}_{-}, a_{+}\right)=0$ has exactly one solution which is the sonic state, i.e. $v=v^{*}$.

3). If $\Psi\left(v^{*} ; \mathbf{V}_{-}, a_{+}\right)>0$, the equation $\Psi\left(v ; \mathbf{V}_{-}, a_{+}\right)=0$ has no solution.

We emphasize that the criterion (3.15) is consistent with the admissibility condition given by Goatin and LeFloch [9, p. $12(\mathrm{H})]$. After we obtained the velocity of the outflow state, the remaining variables of the outflow state such as the density, pressure and sound speed can be calculated from the relations (3.6), (3.7) and (3.8). Therefore, the stationary wave curve in a fixed monotonic duct with $a_{-}$as the inflow area and $a_{+}$as the outflow area can be viewed as the set of the states $\mathbf{J}\left(a ; \mathbf{U}_{-}, a_{-}\right)$, where $a$ varies from $a_{-}$to $a_{+}$.

However, as we have seen in Corollary 3.3 that the velocity function may have no solutions in a fixed duct with certain inflow states. To be more precise we now investigate the existence conditions for the state $\mathbf{J}\left(a_{+} ; \mathbf{U}_{-}, a_{-}\right)$introduced above. The existence condition of the outflow state $\mathbf{U}=\mathbf{J}\left(a_{+} ; \mathbf{U}_{-}, a_{-}\right)$is that the corresponding velocity function $\Psi\left(v ; \mathbf{V}_{-}, a_{+}\right)=0$ has at least one solution. Additionally, Corollary 3.3 reveals that this is equivalent to the minimum value of the velocity function $\Psi\left(v ; \mathbf{V}_{-}, a_{+}\right)$being not larger than 0 , i.e.

$$
\Psi\left(v^{*} ; \mathbf{V}_{-}, a_{+}\right)=\frac{\gamma+1}{2(\gamma-1)} c_{-}^{2}\left(\frac{a_{-} v_{-}}{a_{+} c_{-}}\right)^{\frac{2(\gamma-1)}{\gamma+1}}-\frac{v_{-}^{2}}{2}-\frac{c_{-}^{2}}{\gamma-1} \leq 0 .
$$

Dividing $c_{-}^{2}$ into the relation (3.16) we obtain

$$
\frac{\gamma+1}{2(\gamma-1)}\left(\frac{a_{-} v_{-}}{a_{+} c_{-}}\right)^{\frac{2(\gamma-1)}{\gamma+1}}-\frac{v_{-}^{2}}{2 c_{-}^{2}}-\frac{1}{\gamma-1} \leq 0 .
$$

Define $m_{-}:=M_{-}^{2}=\left(\frac{v_{-}}{c_{-}}\right)^{2}$ as the square of the Mach number $M_{-}$, then the left part of $(3.17)$ is the function

$$
\varphi\left(m_{-} ; a_{-}, a_{+}\right):=\frac{\gamma+1}{2(\gamma-1)}\left(\frac{a_{-}}{a_{+}}\right)^{\frac{2(\gamma-1)}{\gamma+1}} m_{-}^{\frac{\gamma-1}{\gamma+1}}-\frac{m_{-}}{2}-\frac{1}{\gamma-1} .
$$

Obviously we transformed the existence condition of the outflow state $\mathbf{U}=\mathbf{J}\left(a_{+} ; \mathbf{U}_{-}, a_{-}\right)$ into finding the region for $m_{-}$which satisfied $\varphi\left(m_{-} ; a_{-}, a_{+}\right) \leq 0$. We summarize the results in the following theorem.

THEOREM 3.4. The existence condition for the outflow state $\mathbf{U}=\mathbf{J}\left(a_{+} ; \mathbf{U}_{-}, a_{-}\right)$ is as follows,

1. if $\frac{a_{-}}{a_{+}}<1$, the state $\mathbf{U}=\mathbf{J}\left(a_{+} ; \mathbf{U}_{-}, a_{-}\right)$exists for arbitrary $\mathbf{U}_{-}$. 
2. if $\frac{a_{-}}{a_{+}}>1$, the state $\mathbf{U}=\mathbf{J}\left(a_{+} ; \mathbf{U}_{-}, a_{-}\right)$exists iff $m_{-} \leq \beta_{l}<1$ or $m_{-} \geq \beta_{r}$ where the values $\beta_{l}$ and $\beta_{r}$ are the left and right solutions of $\varphi\left(m_{-} ; a_{-}, a_{+}\right)=$ 0 .

Proof. Consider the behavior of the function $\varphi\left(m_{-} ; a_{-}, a_{+}\right)$. It is a continuous function of $m_{-}$and the derivative is

$$
2 \varphi^{\prime}\left(m_{-} ; a_{-}, a_{+}\right)=\left(\frac{a_{-}}{a_{+}}\right)^{\frac{2(\gamma-1)}{\gamma+1}} m_{-}^{\frac{-2}{\gamma+1}}-1 .
$$

So we have

$$
\varphi^{\prime}\left(m_{-} ; a_{-}, a_{+}\right) \begin{cases}>0, & \text { iff } m_{-}<\left(\frac{a_{-}}{a_{+}}\right)^{\gamma-1}, \\ =0, & \text { iff } m_{-}=\left(\frac{a_{-}}{a_{+}}\right)^{\gamma-1}, \\ <0, & \text { iff } m_{-}>\left(\frac{a_{-}}{a_{+}}\right)^{\gamma-1} .\end{cases}
$$

That is to say the function $\varphi\left(m_{-} ; a_{-}, a_{+}\right)$is an increasing function when $m_{-}<$ $\left(\frac{a_{-}}{a_{+}}\right)^{\gamma-1}$ and a decreasing function when $m_{-}>\left(\frac{a_{-}}{a_{+}}\right)^{\gamma-1}$; it reaches the maximum value at $m_{-}^{*}=\left(\frac{a_{-}}{a_{+}}\right)^{\gamma-1}$ and $\varphi\left(m_{-}^{*} ; a_{-}, a_{+}\right)=\frac{\left(\frac{a_{-}}{a_{+}}\right)^{\gamma-1}-1}{\gamma-1}$.

So when $a_{-}<a_{+}$, the value $\varphi\left(m_{-} ; a_{-}, a_{+}\right)<\varphi\left(m_{-}^{*} ; a_{-}, a_{+}\right)<0$ for any $m_{-}$; when $a_{-}>a_{+}$, the maximum values $\varphi\left(m_{-}^{*} ; a_{-}, a_{+}\right)>0$ and the equation $\varphi\left(m_{-} ; a_{-}, a_{+}\right)=0$ has two distinct solutions $\beta_{l}$ and $\beta_{r}$, then the condition for $\varphi\left(m_{-} ; a_{-}, a_{+}\right) \leq 0$ is $m_{-} \leq \beta_{l}$ or $m_{-} \geq \beta_{r}$. Moreover we have $\beta_{l}<1$ since $\varphi\left(1 ; a_{-}, a_{+}\right)>0=\varphi\left(\beta_{l} ; a_{-}, a_{+}\right)$.

Theorem 3.4 indicates that the flow in a converging monotonic duct is quite complex, refer also to Liu [12]. To guarantee the existence of the outflow state of a stationary flow in a converging duct, the square of the Mach number of the inflow states must be less than $\beta_{l}$ or larger than $\beta_{r}$. Of course an iteration method can be used to calculate them, however this is not straightforward in the application. Two critical areas $a_{T}$ and $a_{S}$ are defined as the equivalent condition for estimating whether the outflow states of the stationary wave exist or not. Furthermore, they also play important roles in determining whether certain waves occur or not. The details are stated in the following lemma. We emphasize that the symbol $\mathbf{U}_{c}=\left(\rho_{c}, \rho_{c} v_{c}, \rho_{c} E_{c}\right)$ is to denote the sonic state at which $v_{c}=c_{c}$.

LEMMA 3.5. Assume that the duct is converging and strictly monotonic. For the supersonic inflow state $v_{-}^{2}>c_{-}^{2}$, we define $a_{T}$ as the outflow area at which $\mathbf{U}_{c}=\mathbf{J}\left(a_{T} ; \mathbf{U}_{-}, a_{-}\right)$and $a_{S}$ as the outflow area at which $\mathbf{U}_{c}=\mathbf{J}\left(a_{S} ; S_{k}^{0}\left(\mathbf{U}_{-}\right), a_{-}\right)$, then the following facts hold.

1. The equivalent condition for the existence of the state $\mathbf{U}=\mathbf{J}\left(a_{+} ; \mathbf{U}_{-}, a_{-}\right)$is that $a_{+}>a_{T}$, where $a_{T}$ is given as

$$
a_{T}=a_{-}\left|\frac{v_{-}}{c_{-}}\right|\left[\mu^{2}\left(\frac{v_{-}}{c_{-}}\right)^{2}+\left(1-\mu^{2}\right)\right]^{-\frac{1}{2 \mu^{2}}}
$$

and $|\cdot|$ refers the absolute values. 
2. The equivalent condition for the existence of the state $\mathbf{U}=\mathbf{J}\left(a_{+} ; S_{k}^{0}\left(\mathbf{U}_{-}\right), a_{-}\right)$ is that $a_{+}>a_{S}$, where $a_{S}$ is given as

$$
a_{S}=a_{-}\left|\frac{v_{k}^{0}}{c_{k}^{0}}\right|\left[\mu^{2}\left(\frac{v_{k}^{0}}{c_{k}^{0}}\right)^{2}+\left(1-\mu^{2}\right)\right]^{-\frac{1}{2 \mu^{2}}},
$$

and $\mathbf{U}_{\mathbf{k}}^{\mathbf{0}}=S_{k}^{0}\left(\mathbf{U}_{-}\right)$.

3. One always has $a_{S}>a_{T}$.

Proof. For the supersonic inflow state $v_{-}^{2}>c_{-}^{2}$ and the converging duct with the area $a(x)$, the state $\mathbf{J}\left(a ; \mathbf{U}_{-}, a_{-}\right)$approaches the sonic state along the duct. Denote the limiting state as $\mathbf{U}_{c}=\mathbf{J}\left(a_{T} ; \mathbf{U}_{-}, a_{-}\right)$. Since the outflow is the sonic state, hence Lemma 3.2 indicates that

$$
v_{c}=v^{*}=v_{-}\left(\frac{a_{-}}{a_{T}}\right)^{\frac{\gamma-1}{\gamma+1}}\left(\frac{c_{-}}{v_{-}}\right)^{\frac{2}{\gamma+1}} .
$$

Moreover since $\mathbf{U}_{c}$ is the sonic state, thus (3.8) implies that

$$
v_{c}^{2}=\mu^{2} v_{-}^{2}+\left(1-\mu^{2}\right) c_{-}^{2} .
$$

where $\mu^{2}=\frac{\gamma-1}{\gamma+1}$. We can derive $a_{T}$ in (3.21) by equaling squared (3.23) and (3.24).

In addition the function $\varphi\left(m_{-} ; a_{-}, a_{+}\right)$defined in $(3.18)$ is a decreasing function of the outflow area $a_{+}$. So when $a_{+}>a_{T}, \varphi\left(m_{-}^{2} ; a_{-}, a_{+}\right)<\varphi\left(m_{-}^{2} ; a_{-}, a_{T}\right)=0$. Then according to Corollary 3.3 , there are two distinct solutions to $\Psi\left(v ; \mathbf{V}_{-}, a_{+}\right)=0$. Thus when $a_{+}>a_{T}$, the stationary wave curve $\mathbf{U}=\mathbf{J}\left(a_{+} ; \mathbf{U}_{-}, a_{-}\right)$exists. Similarly the claims with respect of the area $a_{S}$ can be proved analogously.

Now we want to prove $a_{S}>a_{T}$. We only consider the case $k=1$, the other case $k=3$ can be derived in the same way. Since $\sigma_{1}=0$, from (2.8) we obtain

$$
\frac{p_{1}^{0}}{p_{-}}=\left(1+\mu^{2}\right)\left(\frac{v_{-}}{c_{-}}\right)^{2}-\mu^{2}
$$

Using (3.25) in (2.9) and with a short calculation we have

$$
\left\{\begin{array}{l}
v_{1}^{0}=\mu^{2} v_{-}+\left(1-\mu^{2}\right) \frac{c_{-}^{2}}{v_{-}} \\
\left(c_{1}^{0}\right)^{2}=c_{-}^{2}\left[\left(1+\mu^{2}\right)\left(\frac{v_{-}}{c_{-}}\right)-\mu^{2}\left(\frac{c_{-}}{v_{-}}\right)\right]\left[\mu^{2}\left(\frac{v_{-}}{c_{-}}\right)+\left(1-\mu^{2}\right)\left(\frac{c_{-}}{v_{-}}\right)\right] .
\end{array}\right.
$$

Since $m_{-}=\left(\frac{v_{-}}{c_{-}}\right)^{2}$ define $s\left(m_{-}\right)=\left(\frac{v_{1}^{0}}{c_{1}^{0}}\right)^{2}$, then from $(3.26)$ we have

$$
s\left(m_{-}\right)=\frac{\mu^{2} m_{-}+\left(1-\mu^{2}\right)}{\left(1+\mu^{2}\right) m_{-}-\mu^{2}} .
$$

Using (3.27) into (3.21) and (3.22), we define the following function

$$
\begin{aligned}
H\left(m_{-}\right):=\left(\frac{a_{S}}{a_{T}}\right)^{2} & =\frac{s\left(m_{-}\right)\left[\mu^{2} m_{-}+\left(1-\mu^{2}\right)\right]^{\frac{1}{\mu^{2}}}}{m_{-}\left[\mu^{2} s\left(m_{-}\right)+\left(1-\mu^{2}\right)\right]^{\frac{1}{\mu^{2}}}} \\
& =\left(\mu^{2}+\left(1-\mu^{2}\right) m_{-}^{-1}\right)^{1+\frac{1}{\mu^{2}}}\left(\left(1+\mu^{2}\right) m_{-}-\mu^{2}\right)^{\frac{1}{\mu^{2}}-1} .
\end{aligned}
$$

The derivative of the function $H\left(m_{-}\right)$is

$$
H^{\prime}\left(m_{-}\right)=\left(1+\mu^{2}\right)\left(1-\mu^{2}\right)\left(m_{-}^{-1}-1\right)^{2}\left[\mu^{2}+\left(1-\mu^{2}\right) m_{-}^{-1}\right]^{\frac{1}{\mu^{2}}}\left[\left(1+\mu^{2}\right) m_{-}-\mu^{2}\right]^{\frac{1}{\mu^{2}}-2}
$$


So $H^{\prime}\left(m_{-}\right)>0$ when $m_{-}>1$. Thus we have $H\left(m_{-}\right)>H(1)=1$ when $m_{-}>1$, i.e. $a_{S}>a_{T}$.

COROLlary 3.6. Lemma 3.5 shows that if the state $\mathbf{J}\left(a_{+} ; \mathbf{U}_{-}, a_{-}\right)$fails to exist, then $\mathbf{J}\left(a_{+} ; S_{k}^{0}\left(\mathbf{U}_{-}\right), a_{-}\right)$also fails to exist, but the opposite relation is not true.

4. The Riemann problem for Euler equations in a duct with discontinuous cross-section. In this section we solve the Riemann problem for the system (1.6) with the initial data (2.5). Note that $a(x)_{t}=0$, i.e. the duct is independent of the time. Hence the area of the duct satisfies

$$
a(x, t)= \begin{cases}a_{L}, & x<0 \\ a_{R}, & x>0 .\end{cases}
$$

We remark that if $a_{L}=a_{R}$ the system (1.6) totally degenerates into the homogeneous Euler equations (1.4). Its exact solution has been reviewed in Section 2.4. For more details refer to Courant and Friedrichs [4], as well as Toro [19]. Therefore, throughout this paper we only consider cases for which $a_{L} \neq a_{R}$.

Experimental results show that within some short time, the flow near the jump in the cross section becomes stationary, see Dulov [6] cited by Andrianov and Warnecke in [1]. Here we deal with the stationary flows as a limiting case, an approach which was used by Marchesin and Paes-Leme [15]. They regarded the discontinuous diameter duct as the limiting case of a piecewise monotonic duct. In Section 3 we obtained that the stationary flows in the monotonic duct are governed by the stationary wave curve composed of states $\mathbf{J}\left(a ; \mathbf{U}_{-}, a_{-}\right)$, where $a$ varies from $a_{-}$to $a_{+}$. So the stationary wave of the Riemann solution to the system (1.6) can be viewed as a 0 width transition layer located at the initial discontinuity $x=0$.

Therefore, the exact Riemann solution consists of 0 -waves which are stationary waves located at $x=0$, a sequence of 1 - and 3 -shocks or rarefactions, as well as a 2 -wave which is a contact discontinuity. As before we project all states into the $(v, p)$ phase plane and define $1-\mathrm{M}$ curves $T_{1}\left(\mathbf{U}_{L}\right)$ as the set of states which can be connected to the state $\mathbf{U}_{L}$ by the 1 -waves and similarly the $3-\mathrm{M}$ curves $T_{3}\left(\mathbf{U}_{R}\right)$. Note that the mutual positions of the 0 -wave and the other three elementary waves are not known a priori. The resolution of this issue is to merge the 0 -wave curve into the 1 - or $3-\mathrm{M}$ curves and name them $\mathrm{L}-\mathrm{M}$ and $\mathrm{R}-\mathrm{M}$ curves. These two curves can be regarded as an extension of the $1-\mathrm{M}$ curve $T_{1}\left(\mathbf{U}_{L}\right)$ and the $3-\mathrm{M}$ curve $T_{3}\left(\mathbf{U}_{R}\right)$ respectively. They will serve as a basis for the calculation of the Riemann solutions to the duct flow. We neglect the $2-$ waves since the pressure and the velocity are continuous across it. Wave curves play a fundamental role in finding the exact Riemann solutions to the homogeneous Euler equations (1.4), see e.g. Section 2.4. In the next section we present the details of these wave curves.

4.1. Wave curves structure. We define the left wave curve $W_{L}\left(\mathbf{U}_{L}\right)$ as a curve in the $(v, p)$ phase plane starting at the state $\left(v_{L}, p_{L}\right)$ and ending at a state $\left(v_{M}, p_{M}\right)$. The left wave curve $W_{L}\left(\mathbf{U}_{L}\right)$ consists of a continuous succession of components, which include, not necessarily all present, 1-shock curves $S_{1}$, 1-rarefaction curves $R_{1}$, and a stationary wave curve. Similarly, the right wave curve $W_{R}\left(\mathbf{U}_{R}\right)$ is defined in an analogous manner, it starts at $\left(v_{R}, p_{R}\right)$ and ends at $\left(v_{M}, p_{M}\right)$. As in solving the Riemann problem (1.4), cf. Section 2.4, a full wave curve for the Riemann problem (1.6) and (2.5) is a left wave curve $W_{L}\left(\mathbf{U}_{L}\right)$ from $\left(v_{L}, p_{L}\right)$ to $\left(v_{M}, p_{M}\right)$ followed by a right wave curve $W_{R}\left(\mathbf{U}_{R}\right)$ from $\left(v_{M}, p_{M}\right)$ to $\left(v_{R}, p_{R}\right)$. The algorithm for finding the exact solution has two steps: the first is to find $\left(v_{M}, p_{M}\right)$ for the given initial Riemann 
data (2.5). The second is to construct the corresponding left wave curve $W_{L}\left(\mathbf{U}_{L}\right)$ and right wave curve $W_{R}\left(\mathbf{U}_{R}\right)$.

To determine $\left(v_{M}, p_{M}\right)$ for the given states $\mathbf{U}_{L}$ and $\mathbf{U}_{R}$ we define the $\mathrm{L}-\mathrm{M}$ curve $\mathbf{C}_{L}\left(\mathbf{U}_{L}\right)$ and the $\mathrm{R}-\mathrm{M}$ curve $\mathbf{C}_{R}\left(\mathbf{U}_{R}\right)$. The intermediate states $\left(v_{M}, p_{M}\right)$ are the intersection points of the two curves

$$
\begin{aligned}
& \mathbf{C}_{L}\left(\mathbf{U}_{L}\right)=\left\{\mathbf{U} \mid \mathbf{U} \text { is connected to } \mathbf{U}_{L} \text { by a left wave curve } W_{L}\left(\mathbf{U}_{L}\right)\right\}, \\
& \mathbf{C}_{R}\left(\mathbf{U}_{R}\right)=\left\{\mathbf{U} \mid \mathbf{U} \text { is connected to } \mathbf{U}_{R} \text { by a right wave curve } W_{R}\left(\mathbf{U}_{R}\right)\right\} .
\end{aligned}
$$

There is precisely one stationary wave in a full wave curve from $\mathbf{U}_{L}$ to $\mathbf{U}_{R}$ located either on the $\mathrm{L}-\mathrm{M}$ curve or the $\mathrm{R}-\mathrm{M}$ curve. Due to the fact that the velocity is continuous across the contact discontinuity and does not change sign through the stationary wave, the location of the stationary wave is determined by this rule: If $v_{M}>0$ the stationary wave is on the L-M curve; if $v_{M}<0$ the stationary wave is on the $\mathrm{R}-\mathrm{M}$ curve. There are respectively six different types of the $\mathrm{L}-\mathrm{M}$ curves $\mathbf{C}_{L}\left(\mathbf{U}_{L}\right)$ and $\mathrm{R}-\mathrm{M}$ curves $\mathbf{C}_{R}\left(\mathbf{U}_{R}\right)$. We list all cases in Table 1 . In some sense the $\mathrm{R}-\mathrm{M}$ curves

TABLE 4.1

Types of $L-M$ and $R-M$ curves

\begin{tabular}{|l|ll|ll|}
\hline Case & $\mathbf{C}_{L}\left(\mathbf{U}_{L}\right)$ & $\mathbf{C}_{R}\left(\mathbf{U}_{R}\right)$ \\
\hline I & $v_{L}-c_{L}<0 ;$ & $a_{L}>a_{R}$ & $v_{R}+c_{R}>0 ;$ & $a_{R}>a_{L}$ \\
II & $v_{L}-c_{L} \leq 0 ;$ & $a_{L}<a_{R}$ & $v_{R}+c_{R} \geq 0 ;$ & $a_{R}<a_{L}$ \\
III & $v_{L}-c_{L}>0 ;$ & $a_{L}<a_{R}$ & $v_{R}+c_{R}<0 ;$ & $a_{R}<a_{L}$ \\
IV & $v_{L}-c_{L}>0 ;$ & $a_{L}>a_{R}>a_{S}>a_{T}$ & $v_{R}+c_{R}<0 ;$ & $a_{R}>a_{L}>a_{S}>a_{T}$ \\
V & $v_{L}-c_{L}>0 ;$ & $a_{L}>a_{S}>a_{R}>a_{T}$ & $v_{R}+c_{R}<0 ;$ & $a_{R}>a_{S}>a_{L}>a_{T}$ \\
VI & $v_{L}-c_{L}>0 ;$ & $a_{L}>a_{S}>a_{T}>a_{R}$ & $v_{R}+c_{R}<0 ;$ & $a_{R}>a_{S}>a_{T}>a_{L}$ \\
\hline
\end{tabular}

can be regarded as a reflection of the $\mathrm{L}-\mathrm{M}$ curves with respect to the axis $v=0$ in the $(v, p)$ phase space. So in the following we will study all six cases of the $\mathrm{L}-\mathrm{M}$ curves in detail and the $\mathrm{R}-\mathrm{M}$ curves can then be treated likewise. We point out that the classification of the $\mathrm{L}-$ and $\mathrm{R}-\mathrm{M}$ curves is based on the framework given by Marchesin and Paes-Leme in [15] for simple isothermal system.

It turns out that there are two groups of wave configurations. One group is without resonance and the other is with resonance of the waves. The wave configurations of the group without resonance consist of a stationary wave and three elementary waves, while the wave configurations of the group with resonance consist of a resonant wave and two elementary waves. Certainly one can expect that the resonant waves are complicated. Goatin and LeFloch in [9] studied the resonant waves at which the nonlinear characteristic $\lambda_{k}=\lambda_{0}$. We summarize the details of all types of resonant waves in the next section.

Based on the types of the resonant waves and the position of the 1-wave in terms of the 0 -wave, we classify the wave configurations with positive intermediate velocity in Figures 4.1, 4.2, 4.9, 4.10, 4.17, 4.18 and 4.31. Particularly we use the letters $A-H$ to denote them. The wave configurations with negative intermediate velocity can be treated as appropriate symmetric cases of the ones with positive intermediate velocity. The details of all possible wave configurations are included in the construction of the $\mathrm{L}-\mathrm{M}$ curves.

In the next section we construct the $\mathrm{L}-\mathrm{M}$ curve for all cases. Also the construction is validated by a series of examples. Unless otherwise stated, we assume that the space 
variable $x \in[0,2]$ and that the initial discontinuity is located at $x=0.8$.

4.1.1. Case I: $v_{L}-c_{L}<0 ; a_{L}>a_{R}$. In this case the possible wave configurations with positive intermediate velocity are the wave configurations $A$ and $B$, see Figures 4.1 and 4.2. The wave configuration $A$ consists of, from left to right, a 1-wave, a stationary wave located at $x=0$, a contact discontinuity and a 3 -wave. The wave configuration $B$ consists of, from left to right, a resonant wave named $W S W_{1}\left(\mathbf{U}_{L}, a_{L}, a_{R}\right)^{1}$ since the stationary wave coincides with the 1 -wave, a contact discontinuity and a 3-wave. The 1-wave, in such a case, is definitely a transonic rarefaction wave because the 1 -shock has a negative speed due to the Lax condition. Therefore, the resonant wave $W S W_{1}\left(\mathbf{U}_{L}, a_{L}, a_{R}\right)$ here is constituted of two parts, the first part is a rarefaction wave along $T_{1}\left(\mathbf{U}_{L}\right)$ from the state $\mathbf{U}_{L}$ to the state $\tilde{\mathbf{U}}_{c}$; the second part is the 1 -wave along $T_{1}\left(\mathbf{U}_{c}\right)$. These two parts are separated by an intermediate constant state.

Consequently, the $\mathrm{L}-\mathrm{M}$ curve $C_{L}\left(\mathbf{U}_{L}\right)$ consists of three parts

$$
\begin{aligned}
& Q_{1}\left(\mathbf{U}_{L}\right)=\left\{\mathbf{U} \mid \mathbf{U} \in T_{1}\left(\mathbf{U}_{L}\right) \text { with } v \leq 0\right\}, \\
& Q_{2}\left(\mathbf{U}_{L}\right)=\left\{\mathbf{U} \mid \mathbf{U}=\mathbf{J}\left(a_{R} ; \mathbf{U}_{-}, a_{L}\right) \text { and } \mathbf{U}_{-} \in T_{1}\left(\mathbf{U}_{L}\right) \text { with } 0<v_{-}<\tilde{v}_{c}, 0<v<v_{c}\right\}, \\
& Q_{3}\left(\mathbf{U}_{L}\right)=\left\{\mathbf{U} \mid \mathbf{U} \in T_{1}\left(\mathbf{U}_{c}\right) \text { with } v>v_{c}\right\},
\end{aligned}
$$

where $\mathbf{U}_{c}=\mathbf{J}\left(a_{R} ; \tilde{\mathbf{U}}_{c}, a_{L}\right)$, and $\tilde{\mathbf{U}}_{c} \in T_{1}\left(\mathbf{U}_{L}\right)$. The first part $Q_{1}\left(\mathbf{U}_{L}\right)$ is the curve $T_{1}\left(\mathbf{U}_{L}\right)$ which corresponds to negative velocity.

Obviously, the L-M curve $C_{L}\left(\mathbf{U}_{L}\right)=\bigcup_{k=1}^{3} Q_{k}\left(\mathbf{U}_{L}\right)$ is continuous. From Lemma A.1, the part $Q_{2}\left(\mathbf{U}_{L}\right)$ is decreasing in the $(v, p)$ plane. Note that $Q_{k}\left(\mathbf{U}_{L}\right), k=1,3$ are also decreasing. Therefore, the $\mathrm{L}-\mathrm{M}$ curve $C_{L}\left(\mathbf{U}_{L}\right)$ is continuous and decreasing in the $(v, p)$ plane, see Figures 4.3 and 4.6.

If $\left(v_{M}, p_{M}\right) \in Q_{2}\left(\mathbf{U}_{L}\right)$, the wave configuration is shown in Figure 4.1. Obviously, it is classical and has been studied by Andrianov and Warnecke in [1]. Since $a_{L}>a_{R}$, according to Proposition 3.1 the part $Q_{2}\left(\mathbf{U}_{L}\right)$ has $v$ between 0 and $v_{c}$. As an example, we use the Riemann initial data given in Table 4.2 to illustrate the exact solution. The corresponding wave curve is shown in Figure 4.3. The density and velocity of the exact solution at $t=10 e-4 s$ are shown in Figures 4.4 and 4.5.

TABLE 4.2

The Riemann initial data for $\left(v_{M}, p_{M}\right) \in Q_{2}\left(\mathbf{U}_{L}\right)$ in Case $\mathbf{I}$.

\begin{tabular}{|l|l|l|l|l|}
\hline & $a(x)$ & $\rho\left(\mathrm{kg} \cdot \mathrm{m}^{-3}\right)$ & $v\left(\mathrm{~m} \cdot \mathrm{s}^{-1}\right)$ & $p(P a)$ \\
\hline$V_{L}$ & 1.0 & 3.6 & 0.0 & 400000 \\
\hline$V_{R}$ & 0.6 & 0.570370 & 329.130629 & 200000 \\
\hline
\end{tabular}

If $\left(v_{M}, p_{M}\right) \in Q_{3}\left(\mathbf{U}_{L}\right)$, the wave configuration is given in Figure 4.2. We take the Riemann initial data used by Rochette et al. [16] listed in Table 4.3 to illustrate the exact solution. The corresponding wave curves are shown in Figure 4.6. The density and velocity of the exact solution at $t=12 e-4 s$ are shown in Figures 4.7 and 4.8.

4.1.2. Case II: $v_{L} \leq c_{L} ; a_{L}<a_{R}$. In this case the possible wave configurations with positive intermediate velocity are the wave configurations $A, C$ and $D$, see Figures $4.1,4.9$ and 4.10 . The wave configuration $C$ consists of, from left to right, a res-

\footnotetext{
${ }^{1}$ The notation $W S W_{1}$ means that the wave is composed from left to right of a 1 -wave $W$, a stationary wave $S$, and another 1 -wave $W$. Of course the 1 -wave $W$ may be a shock or rarefaction wave. The subscript 1 means that all rarefactions or shocks are related to the 1 -family.
} 


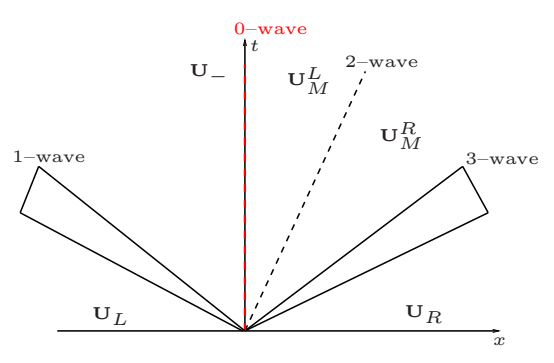

FIG. 4.1. Wave configuration A

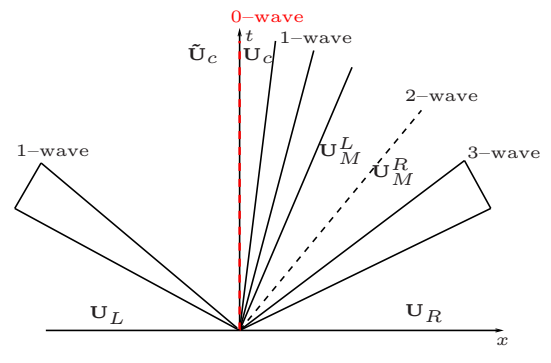

FIG. 4.2. Wave configuration $B$

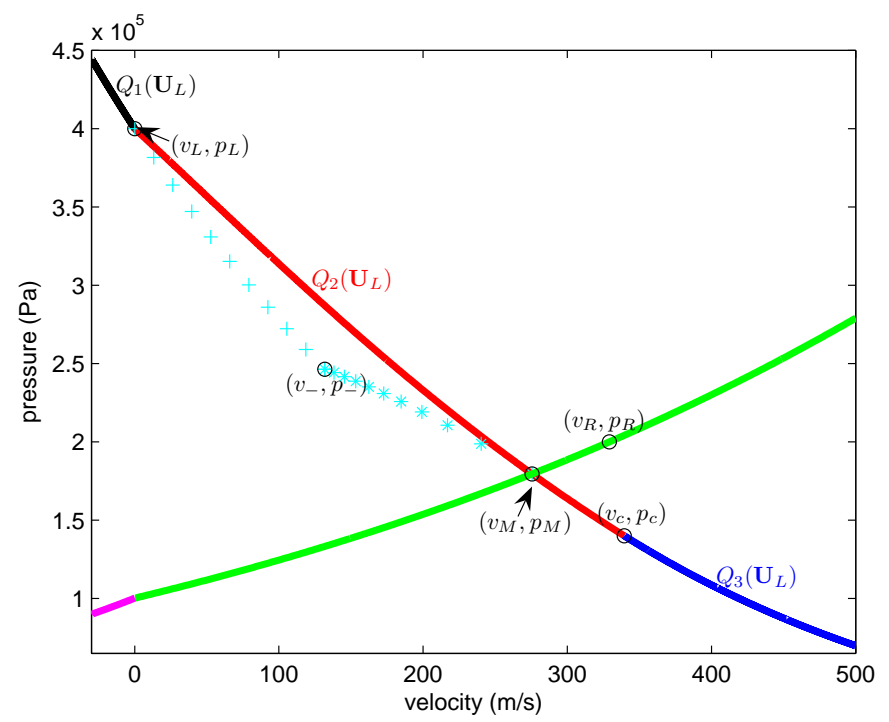

FIG. 4.3. Wave curves for the Riemann initial data in Table 4.2. The $L-M$ curve $C_{L}\left(\mathbf{U}_{L}\right)=$ $\bigcup^{3} Q_{k}\left(\mathbf{U}_{L}\right)$. The left wave curve $W_{L}\left(\mathbf{U}_{L}\right)$ is the rarefaction from $\left(v_{L}, p_{L}\right)$ to $\left(v_{-}, p_{-}\right)($cyan +$)$, followed by the stationary wave from $\left(v_{-}, p_{-}\right)$to $\left(v_{M}, p_{M}\right)($ cyan $*)$. The right wave curve $W_{R}\left(\mathbf{U}_{R}\right)$ is the 3-wave from $\left(v_{R}, p_{R}\right)$ to $\left(v_{M}, p_{M}\right)$ (green line).

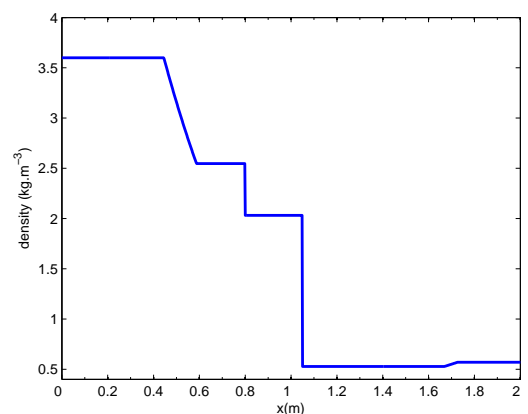

FIG. 4.4. Density for the data in Table 4.2.

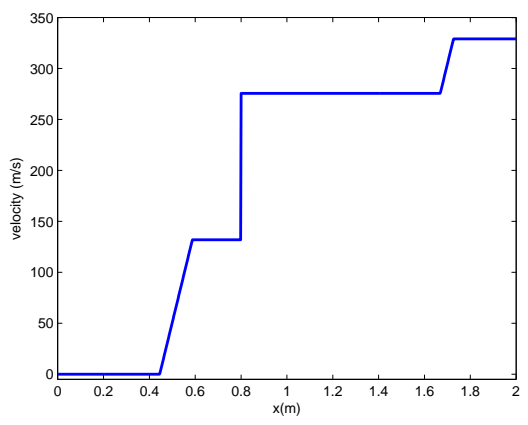

FIG. 4.5. Velocity for the data in Table 4.2. 
TABLE 4.3

The Riemann initial data for $\left(v_{M}, p_{M}\right) \in Q_{3}\left(\mathbf{U}_{L}\right)$ in Case $\mathbf{I}$.

\begin{tabular}{|l|l|l|l|l|}
\hline & $a(x)$ & $\rho\left(\mathrm{kg} \cdot \mathrm{m}^{-3}\right)$ & $v\left(\mathrm{~m} \cdot \mathrm{s}^{-1}\right)$ & $p(P a)$ \\
\hline$V_{L}$ & 1.0 & 3.6 & 0.0 & 400000 \\
\hline$V_{R}$ & 0.6 & 0.570370 & 329.130629 & 75000 \\
\hline
\end{tabular}

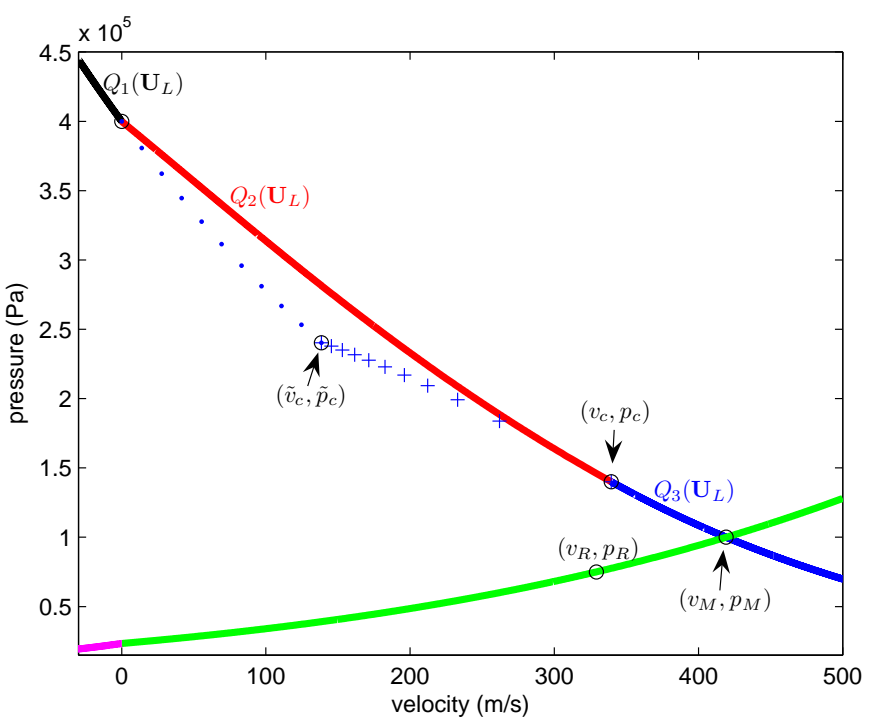

FIG. 4.6. Wave curves for the Riemann initial data in Table 4.3. The left wave curve $W_{L}\left(\mathbf{U}_{L}\right)$ is the rarefaction from $\left(v_{L}, p_{L}\right)$ to $\left(\tilde{v}_{c}, \tilde{p}_{c}\right)$ (blue $\left.\cdot\right)$, followed by the stationary wave from $\left(\tilde{v}_{c}, \tilde{p}_{c}\right)$ to $\left(v_{c}, p_{c}\right)$ $($ blue +$)$, ended by the rarefaction wave from $\left(v_{c}, p_{c}\right)$ to $\left(v_{M}, p_{M}\right)$ (blue line). The right wave curve $W_{R}\left(\mathbf{U}_{R}\right)$ is the $3-$ wave from $\left(v_{R}, p_{R}\right)$ to $\left(v_{M}, p_{M}\right)$ (green line).

onant wave named $R S 0 S_{1}\left(\mathbf{U}_{L}, a_{L}, a_{R}\right)^{2}$ since the stationary wave coincides with the transonic rarefaction wave, a contact discontinuity and a 3 -wave. The resonant waves $R S 0 S_{1}\left(\mathbf{U}_{L}, a_{L}, a_{R}\right)$ is constituted of a rarefaction wave along $T_{1}\left(\mathbf{U}_{L}\right)$ fanning from the state $\mathbf{U}_{L}$ to the sonic state $\mathbf{U}_{c}$ which is defined as $\mathbf{U}_{c}=T_{1}\left(\mathbf{U}_{L}\right) \cap\{\mathbf{U} \mid v=c\}$, followed by a succession of three waves: a supersonic stationary wave $\mathbf{U}_{-}=\mathbf{J}\left(a ; \mathbf{U}_{c}, a_{L}\right)$, a 0 speed 1-shock wave $\mathbf{U}_{+}=S_{1}^{0}\left(\mathbf{U}_{-}\right)$and a subsonic stationary wave $\mathbf{U}=\mathbf{J}\left(a_{R} ; \mathbf{U}_{+}, a\right)$, where the intermediate area $a$ is the location of the zero speed shock. For details concerning the parameter $a$ refers to Marchesin and Paes-Leme [15, p. 446, Fig. 14.]. All of the three waves coalesce on the line $x=0$. The wave configuration $D$ consists of, from left to right, also a resonant wave named $R S W_{1}\left(\mathbf{U}_{L}, a_{L}, a_{R}\right)$, a contact discontinuity and a 3-wave. We study the resonant wave $R S W_{1}\left(\mathbf{U}_{L}, a_{L}, a_{R}\right)$ later for the lack of some essential facts at the moment.

Consequently, the L-M curve $C_{L}\left(\mathbf{U}_{L}\right)$ consists of four parts: $Q_{1}\left(\mathbf{U}_{L}\right), Q_{2}\left(\mathbf{U}_{L}\right)$, $Q_{3}\left(\mathbf{U}_{L}\right)$ and $Q_{4}\left(\mathbf{U}_{L}\right)$. The first two parts $Q_{1}\left(\mathbf{U}_{L}\right)$ and $Q_{2}\left(\mathbf{U}_{L}\right)$ are defined analo-

${ }^{2} R S 0 S_{1}$ means that the wave is composed from left to right of a transonic rarefaction wave $R$, a stationary wave $S$, a 0 -speed 1 -shock 0 , and another stationary wave $S$. The subscript 1 means that all rarefactions or shocks are related to the 1-family. Further down other analogous notation appears. They are used only for configurations of the resonant waves. 


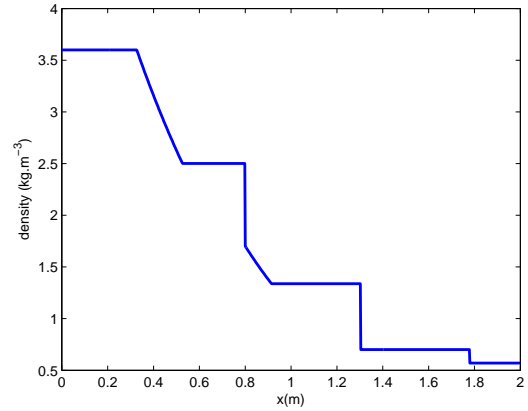

FIG. 4.7. Density for the data in Table 4.3.

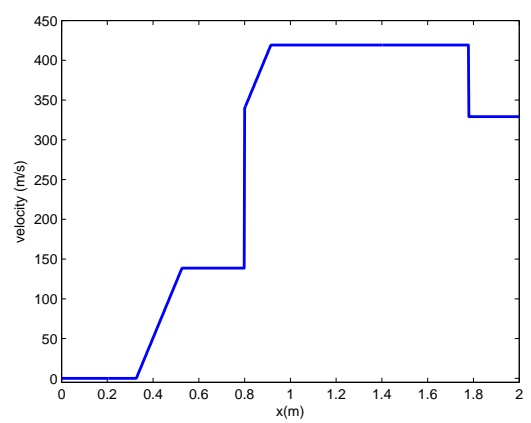

FIG. 4.8. Velocity for the data in Table 4.3.

gously to the Case I

$$
\begin{aligned}
& Q_{1}\left(\mathbf{U}_{L}\right)=\left\{\mathbf{U} \mid \mathbf{U} \in T_{1}\left(\mathbf{U}_{L}\right) \text { with } v<0\right\} \\
& Q_{2}\left(\mathbf{U}_{L}\right)=\left\{\mathbf{U} \mid \mathbf{U}=\mathbf{J}\left(a_{R} ; \mathbf{U}_{-}, a_{L}\right) \text { and } \mathbf{U}_{-} \in T_{1}\left(\mathbf{U}_{L}\right) \text { with } 0<v_{-}<v_{c}, 0<v<\bar{v}_{c}\right\} .
\end{aligned}
$$

Due to $a_{L}>a_{R}$ according to Proposition 3.1, the part $Q_{2}\left(\mathbf{U}_{L}\right)$ has $v \in\left[0, \bar{v}_{c}\right]$, where $\overline{\mathbf{U}}_{c}=\mathbf{J}\left(a_{R} ; \mathbf{U}_{c}, a_{L}\right)$. However, as we have mentioned in Corollary 3.3, if the sonic state $\mathbf{U}_{c}$ is the inflow state of the stationary wave, there may be two solutions, one is subsonic and the other is supersonic, to the velocity function (3.11). Here we use $\overline{\mathbf{U}}_{c}=\mathbf{J}\left(a_{R} ; \mathbf{U}_{c}, a_{L}\right)$ and $\overline{\mathbf{U}}_{c}^{*}=\mathbf{J}\left(a_{R} ; \mathbf{U}_{c}, a_{L}\right)$ to denote the subsonic one and the supersonic one respectively. Note that $Q_{2}\left(\mathbf{U}_{L}\right)$ ends at the subsonic state $\overline{\mathbf{U}}_{c}$.

The third part $Q_{3}\left(\mathbf{U}_{L}\right)$ starts at $\overline{\mathbf{U}}_{c}$ and ends at the $\hat{\mathbf{U}}_{c}=S_{1}^{0}\left(\overline{\mathbf{U}}_{c}^{*}\right)$. It consists of states from the resonant waves $R S 0 S_{1}\left(\mathbf{U}_{L}, a_{L}, a_{R}\right)$. Specifically we have

$$
Q_{3}\left(\mathbf{U}_{L}\right)=\left\{\mathbf{U} \mid \mathbf{U}=\mathbf{J}\left(a_{R} ; \mathbf{U}_{+}, a\right) ; \mathbf{U}_{+}=S_{1}^{0}\left(\mathbf{U}_{-}\right) ; \mathbf{U}_{-}=\mathbf{J}\left(a ; \mathbf{U}_{c}, a_{L}\right), a_{L} \leq a \leq a_{R}\right\}
$$

The last part $Q_{4}\left(\mathbf{U}_{L}\right)$ consists of states from the resonant wave $R S W_{1}\left(\mathbf{U}_{L}, a_{L}, a_{R}\right)$, which is constituted of a rarefaction wave along $T_{1}\left(\mathbf{U}_{L}\right)$ fanning from the state $\mathbf{U}_{L}$ to the state $\mathbf{U}_{c}$, followed by a supersonic stationary wave $\overline{\mathbf{U}}_{c}^{*}=\mathbf{J}\left(a_{R} ; \mathbf{U}_{c}, a_{L}\right)$, and closed by a $1-$ wave $T_{1}\left(\overline{\mathbf{U}}_{c}^{*}\right)$. Thus

$$
Q_{4}\left(\mathbf{U}_{L}\right)=\left\{\mathbf{U} \mid \mathbf{U} \in T_{1}\left(\overline{\mathbf{U}}_{c}^{*}\right) \text { with } v>\hat{\bar{v}}_{c}\right\} .
$$

From Corollary A.4, the segment $Q_{3}\left(\mathbf{U}_{L}\right)$ as a function of the area $a$ is continuous and decreasing. Consequently, the $\mathrm{L}-\mathrm{M}$ wave curve $C_{L}\left(\mathbf{U}_{L}\right)=\bigcup_{k=1}^{4} Q_{k}\left(\mathbf{U}_{L}\right)$ is a continuous and decreasing curve in the $(v, p)$ plane, see Figures 4.11 and 4.14.

If $\left(v_{M}, p_{M}\right)$ belongs to $Q_{2}\left(\mathbf{U}_{L}\right)$, the wave configuration is shown in Figure 4.1.

If $\left(v_{M}, p_{M}\right)$ belongs to $Q_{3}\left(\mathbf{U}_{L}\right)$, the wave configuration is shown in Figure 4.9. We use the Riemann initial data listed in Table 4.4 to illustrate the exact solution at $t=0.5 \mathrm{~s}$. The results are given in Figures 4.11, 4.12 and 4.13.

If $\left(v_{M}, p_{M}\right)$ belongs to $Q_{4}\left(\mathbf{U}_{L}\right)$, the wave configuration is shown in Figure 4.10. The Riemann initial data, as an example, is listed in Table 4.5. The corresponding wave curve and the exact solution at $t=12 e-4 s$ are shown in Figures 4.14, 4.15 and 4.16. Both of these two Riemann initial data were given by Rochette et al. in [16]. 


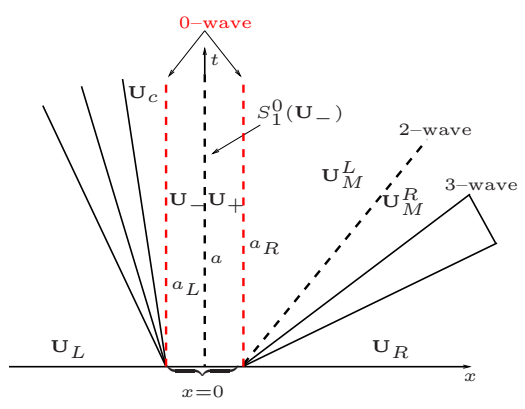

FIG. 4.9. Wave configuration $C$

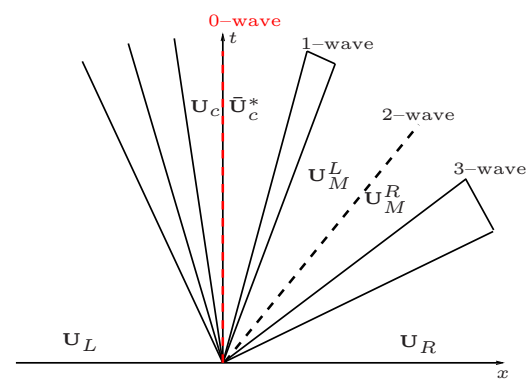

FIG. 4.10. Wave configuration D

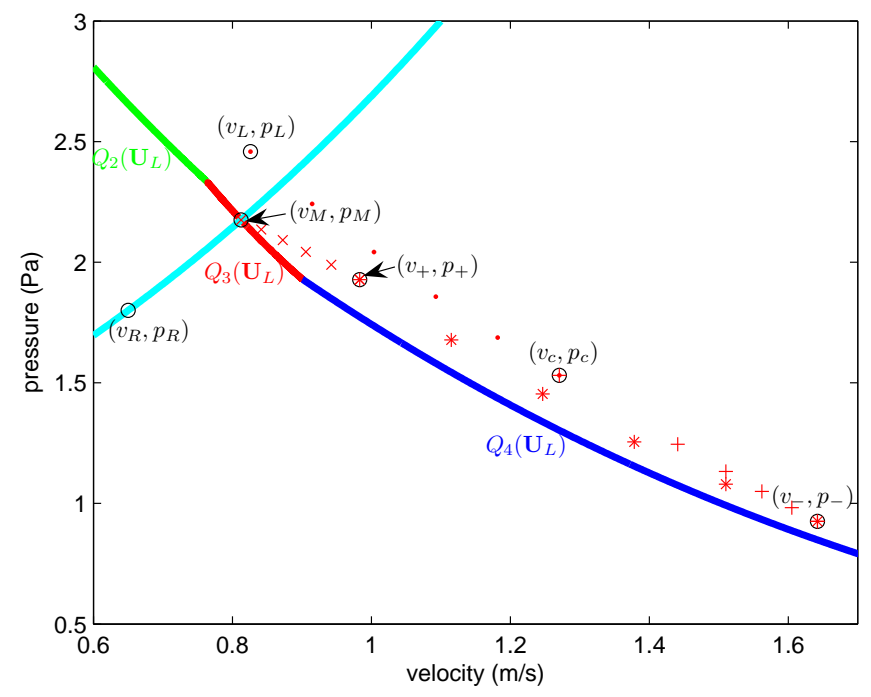

FIG. 4.11. Wave curves for the Riemann initial data in Table 4.4. The left wave curve $W_{L}\left(\mathbf{U}_{L}\right)$ is the rarefaction from $\left(v_{L}, p_{L}\right)$ to $\left(v_{c}, p_{c}\right)$ (red $\left.\cdot\right)$, followed first by the supersonic stationary wave from $\left(v_{c}, p_{c}\right)$ to $\left(v_{-}, p_{-}\right)($red +$)$, then the 0-speed 1-shock from $\left(v_{-}, p_{-}\right)$to $\left(v_{+}, p_{+}\right)($red $*)$, ended by the subsonic stationary wave from $\left(v_{+}, p_{+}\right)$to $\left(v_{M}, p_{M}\right)($ red $\times)$. The right wave curve $W_{R}\left(\mathbf{U}_{R}\right)$ is the 3-shock wave jump from $\left(v_{R}, p_{R}\right)$ to $\left(v_{M}, p_{M}\right)$ (cyan line).

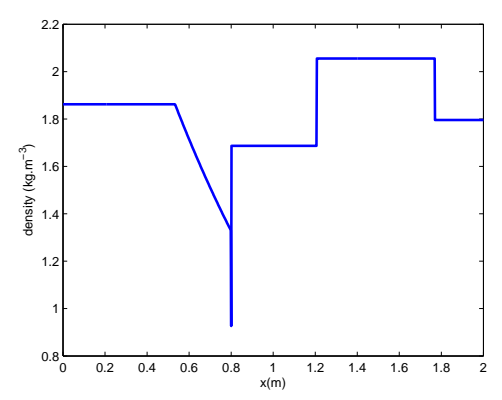

FIG. 4.12. Density for the data in Table 4.4.

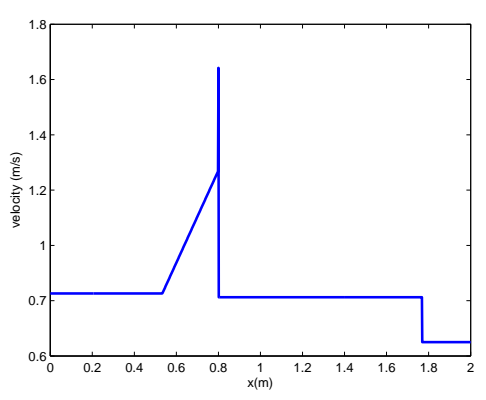

FIG. 4.13. Velocity for the data in Table 4.4. 


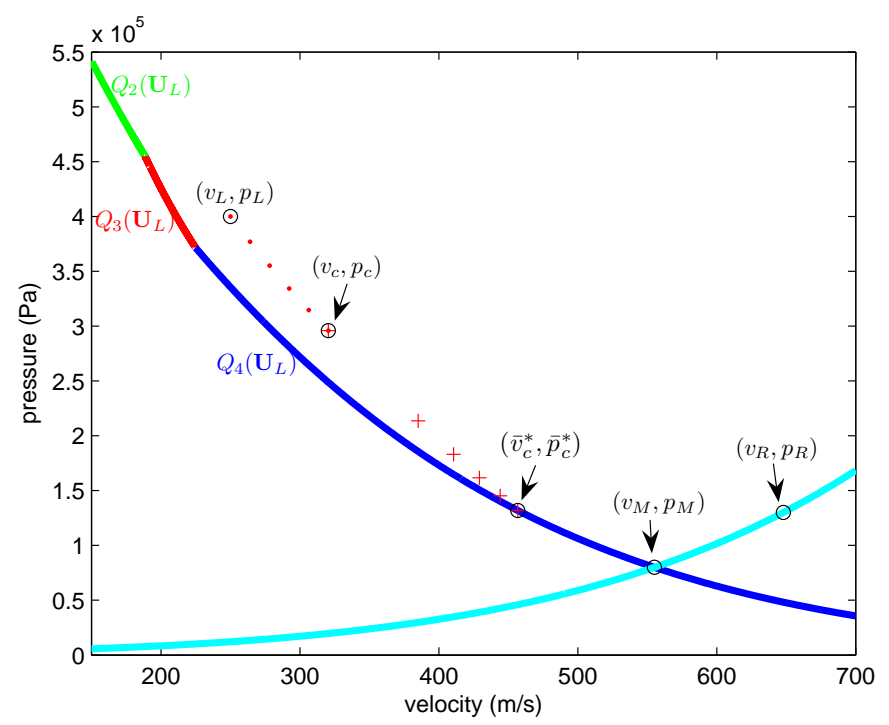

FIG. 4.14. Wave curves for the Riemann initial data in Table 4.5. The left wave curve $W_{L}\left(\mathbf{U}_{L}\right)$ is the rarefaction from $\left(v_{L}, p_{L}\right)$ to $\left(v_{c}, p_{c}\right)$ (blue $\left.\cdot\right)$, followed by the supersonic stationary wave from $\left(v_{c}, p_{c}\right)$ to $\left(\bar{v}_{c}^{*}, \bar{p}_{c}^{*}\right)$ (blue +$)$, ended by the 1-wave from $\left(\bar{v}_{c}^{*}, \bar{p}_{c}^{*}\right)$ to $\left(v_{M}, p_{M}\right)$ (blue line). The right wave curve $W_{R}\left(\mathbf{U}_{R}\right)$ is the 3 -wave from $\left(v_{R}, p_{R}\right)$ to $\left(v_{M}, p_{M}\right)$ (cyan line).

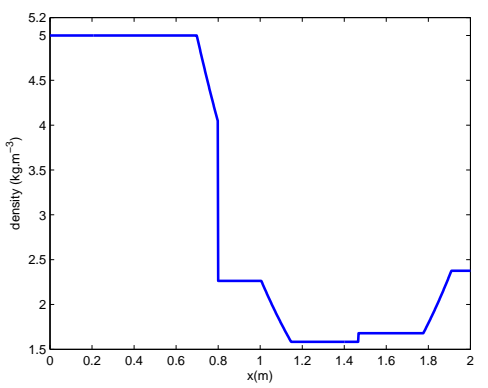

FIG. 4.15. Density for the data in Table 4.5.

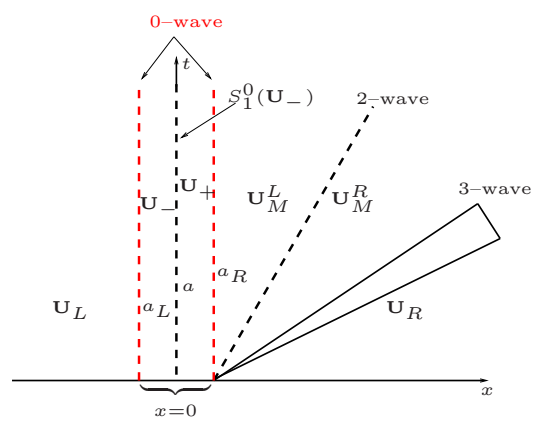

FIG. 4.17. Wave configuration: $E$

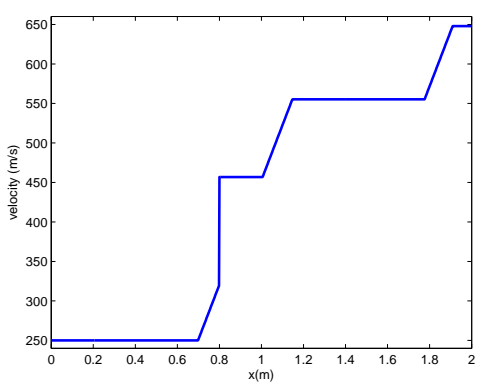

FIG. 4.16. Velocity for the data in Table 4.5.

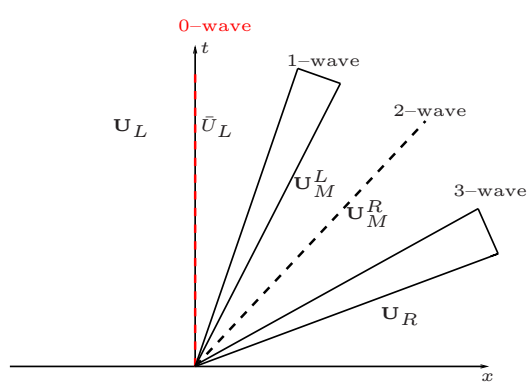

FIG. 4.18. Wave configuration F 
TABLE 4.4

The Riemann initial data for $\left(v_{M}, p_{M}\right) \in Q_{3}\left(\mathbf{U}_{L}\right)$ in Case II.

\begin{tabular}{|l|l|l|l|l|}
\hline & $a(x)$ & $\rho\left(\mathrm{kg} \cdot \mathrm{m}^{-3}\right)$ & $v\left(\mathrm{~m} \cdot \mathrm{s}^{-1}\right)$ & $p(P a)$ \\
\hline$V_{L}$ & 1.3 & 1.862 & 0.826 & 2.4583 \\
\hline$V_{R}$ & 1.6 & 1.795636 & 0.65 & 1.8 \\
\hline
\end{tabular}

TABLE 4.5

The Riemann initial data for $\left(v_{M}, p_{M}\right) \in Q_{4}\left(\mathbf{U}_{L}\right)$ in Case II.

\begin{tabular}{|l|l|l|l|l|}
\hline & $a(x)$ & $\rho\left(\mathrm{kg} \cdot \mathrm{m}^{-3}\right)$ & $v\left(\mathrm{~m} \cdot \mathrm{s}^{-1}\right)$ & $p(P a)$ \\
\hline$V_{L}$ & 0.8 & 5.0 & 250 & 400000 \\
\hline$V_{R}$ & 1.0 & 2.37639 & 647.909308 & 130000 \\
\hline
\end{tabular}

4.1.3. Case III: $v_{L}>c_{L} ; a_{L}<a_{R}$. In this case the possible wave configurations with positive intermediate velocity are the wave configurations $A, E$ and $F$, see Figures 4.1, 4.17 and 4.18. The wave configuration $E$ consists of, from left to right, a resonant wave named $S 0 S_{1}\left(\mathbf{U}_{L}, a_{L}, a_{R}\right)$ since the stationary wave coincides with the 0 -speed 1 -shock wave, a contact discontinuity and a 3 -wave. The resonant wave $S 0 S_{1}\left(\mathbf{U}_{L}, a_{L}, a_{R}\right)$ is constituted of a succession of three waves: a supersonic stationary wave $\mathbf{U}_{-}=\mathbf{J}\left(a ; \mathbf{U}_{L}, a_{L}\right)$, a 0 -speed 1-shock wave $\mathbf{U}_{+}=S_{1}^{0}\left(\mathbf{U}_{-}\right)$and a subsonic stationary wave $\mathbf{U}=\mathbf{J}\left(a_{R} ; \mathbf{U}_{+}, a\right)$, where the intermediate area $a$, as in Case II, denotes the location of the zero speed 1 -shock. The wave configuration $F$ consists of, from left to right, a stationary wave located at $x=0$, a positive 1 -wave, a contact discontinuity and a 3 -wave. Obviously, the wave configuration $F$ is classical and has been studied by Andrianov and Warnecke in [1].

Consequently, the $\mathrm{L}-\mathrm{M}$ curve $C_{L}\left(\mathbf{U}_{L}\right)$ involves four parts similar to Case II. But in contrast to the previous $v_{L} \leq c_{L}$, now $v_{L}>c_{L}$, i.e. the only possible negative 1 -wave is the negative 1-shock. In general, the four parts are defined as follows

$$
\begin{aligned}
Q_{1}\left(\mathbf{U}_{L}\right) & =\left\{\mathbf{U} \mid \mathbf{U} \in T_{1}\left(\mathbf{U}_{L}\right) \text { with } v<0\right\}, \\
Q_{2}\left(\mathbf{U}_{L}\right) & =\left\{\mathbf{U} \mid \mathbf{U}=\mathbf{J}\left(a_{R} ; \mathbf{U}_{-}, a_{L}\right) \text { and } \mathbf{U}_{-} \in S_{1}^{-}\left(\mathbf{U}_{L}\right) \text { with } 0<v_{-}<\hat{v}_{L}, 0<v<\bar{v}_{L}\right\}, \\
Q_{3}\left(\mathbf{U}_{L}\right) & =\left\{\mathbf{U} \mid \mathbf{U}=\mathbf{J}\left(a_{R} ; \mathbf{U}_{+}, a\right) ; \mathbf{U}_{+}=S_{1}^{0}\left(\mathbf{U}_{-}\right) ; \mathbf{U}_{-}=\mathbf{J}\left(a ; \mathbf{U}_{L}, a_{L}\right), a_{L} \leq a \leq a_{R}\right\}, \\
Q_{4}\left(\mathbf{U}_{L}\right) & =\left\{\mathbf{U} \mid \mathbf{U} \in T_{1}\left(\overline{\mathbf{U}}_{L}\right) \text { with } v>\hat{v}_{L}\right\},
\end{aligned}
$$

where $\overline{\mathbf{U}}_{L}=\mathbf{J}\left(a_{R} ; \hat{\mathbf{U}}_{L}, a_{L}\right)$ and $\hat{\mathbf{U}}_{L}=S_{1}^{0}\left(\mathbf{U}_{L}\right)$, while $\hat{\mathbf{U}}_{L}=S_{1}^{0}\left(\overline{\mathbf{U}}_{L}\right)$ and $\overline{\mathbf{U}}_{L}=$ $\mathbf{J}\left(a_{R} ; \mathbf{U}_{L}, a_{L}\right)$. Analogously, the curve $C_{L}\left(\mathbf{U}_{L}\right)$ in this case is also a continuous and decreasing curve in the $(v, p)$ plane.

If the state $\left(v_{M}, p_{M}\right) \in Q_{2}\left(\mathbf{U}_{L}\right)$, the wave configuration is shown in Figure 4.1. One should keep in mind that in this case the 1-wave is a negative shock.

If the intermediate state $\left(v_{M}, p_{M}\right)$ belongs to the segment $Q_{3}\left(\mathbf{U}_{L}\right)$, the wave configuration is shown in Figure 4.17. The Riemann initial data, as an example for this case, is listed in Table 4.6 used by Rochette et al. in [16]. The wave curve and

TABLE 4.6

The Riemann initial data for $\left(v_{M}, p_{M}\right) \in Q_{3}\left(\mathbf{U}_{L}\right)$ in Case III.

\begin{tabular}{|l|l|l|l|l|}
\hline & $a(x)$ & $\rho\left(\mathrm{kg} \cdot \mathrm{m}^{-3}\right)$ & $v\left(\mathrm{~m} \cdot \mathrm{s}^{-1}\right)$ & $p(P a)$ \\
\hline$V_{L}$ & 0.5 & 1.0 & 500 & 100000 \\
\hline$V_{R}$ & 1.0 & 1.34771 & 314.46597 & 250000 \\
\hline
\end{tabular}

the exact solution at $t=12 e-4 s$ of the density and velocity are given in Figures 


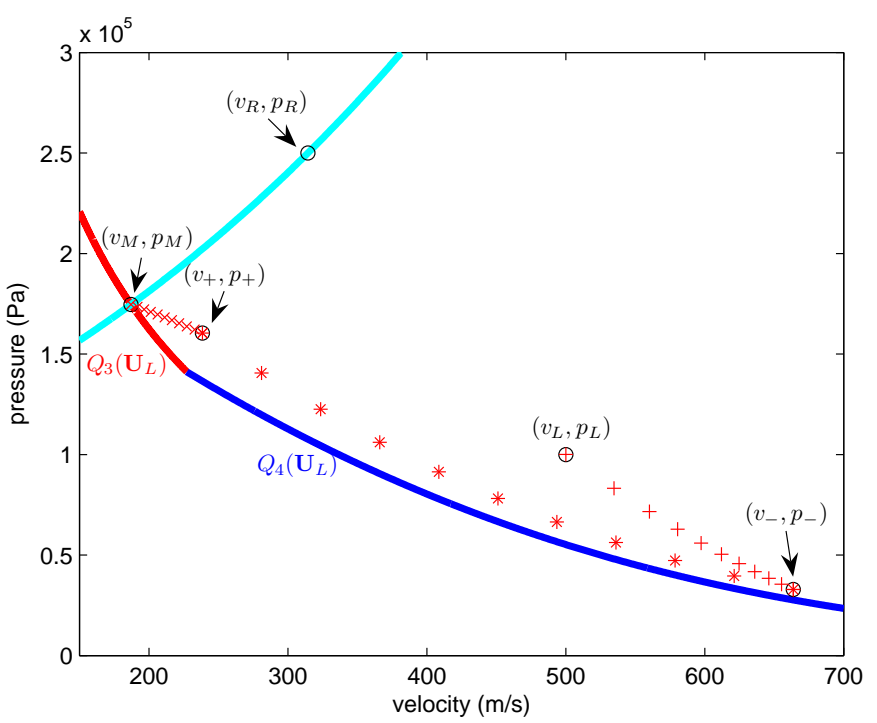

FIG. 4.19. Wave curves for the Riemann initial data in Table 4.6. The left wave curve $W_{L}\left(\mathbf{U}_{L}\right)$ is the stationary wave from $\left(v_{L}, p_{L}\right)$ to $\left(v_{-}, p_{-}\right)($red +$)$, then the 0-speed 1 -shock from $\left(v_{-}, p_{-}\right)$to $\left(v_{+}, p_{+}\right)$(red $\left.*\right)$, ended by the stationary wave from $\left(v_{+}, p_{+}\right)$to $\left(v_{M}, p_{M}\right)($ red $\times)$. The right wave curve $W_{R}\left(\mathbf{U}_{R}\right)$ is the 3 -wave from $\left(v_{R}, p_{R}\right)$ to $\left(v_{M}, p_{M}\right)$ (cyan line).

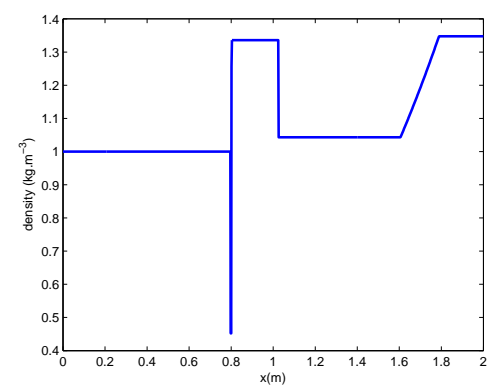

FIG. 4.20. Density for the data in Table 4.6.

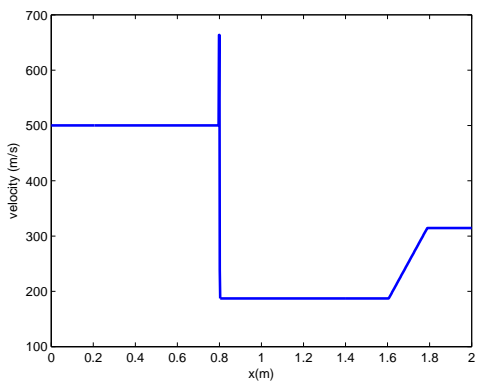

FIG. 4.21. Velocity for the data in Table 4.6.

$4.19,4.20$ and 4.21 .

Finally if $\left(v_{M}, p_{M}\right) \in Q_{4}\left(\mathbf{U}_{L}\right)$, the wave configuration is shown in Figure 4.18. We use the Riemann initial data listed in Table 4.7 for an example of a solution. The

\section{TABLE 4.7}

The Riemann initial data for $\left(v_{M}, p_{M}\right) \in Q_{3}\left(\mathbf{U}_{L}\right)$ in Case $\mathbf{I I I}$.

\begin{tabular}{|l|l|l|l|l|}
\hline & $a(x)$ & $\rho\left(\mathrm{kg} \cdot \mathrm{m}^{-3}\right)$ & $v\left(\mathrm{~m} \cdot \mathrm{s}^{-1}\right)$ & $p(P a)$ \\
\hline$V_{L}$ & 0.5 & 1.0 & 500 & 100000 \\
\hline$V_{R}$ & 1.0 & 1.34771 & 414.46597 & 150000 \\
\hline
\end{tabular}

results are presented in Figures 4.22, 4.23 and 4.24.

4.1.4. Case IV: $v_{L}>c_{L} ; a_{L}>a_{R}>a_{S}>a_{T}$. In this case the wave configurations with positive intermediate velocity are also the wave configurations $A, E$ and 


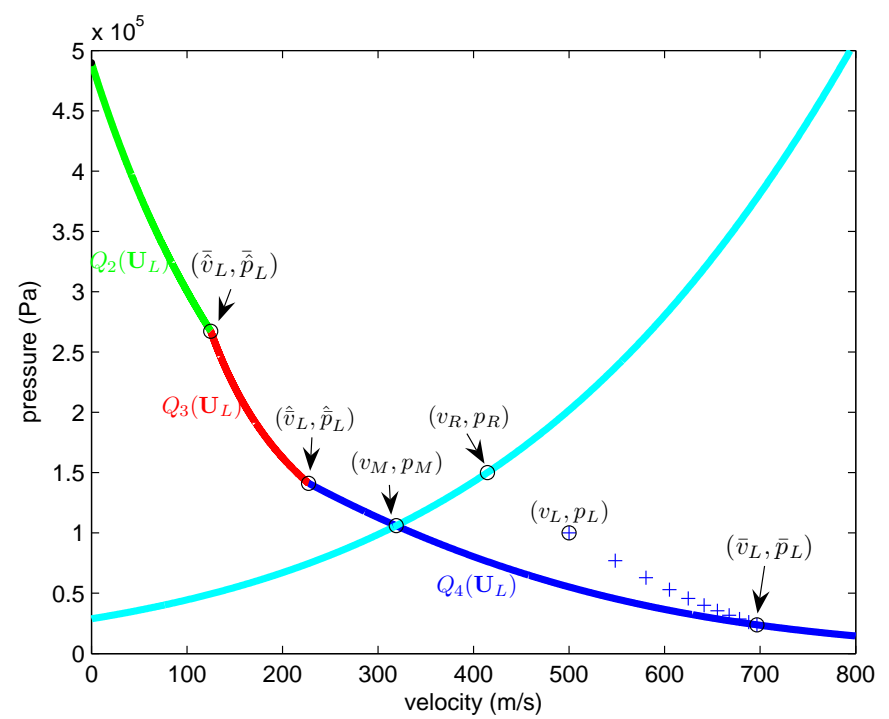

FIG. 4.22. Wave curves for the Riemann initial data in Table 4.7. The left wave curve $W_{L}\left(\mathbf{U}_{L}\right)$ is the stationary wave from $\left(v_{L}, p_{L}\right)$ to $\left(\bar{v}_{L}, \bar{p}_{L}\right)$ (blue +$)$, then the 1 -wave from $\left(\bar{v}_{L}, \bar{p}_{L}\right)$ to $\left(v_{M}, p_{M}\right)$ (blue line). The right wave curve $W_{R}\left(\mathbf{U}_{R}\right)$ is the 3-wave from $\left(v_{R}, p_{R}\right)$ to $\left(v_{M}, p_{M}\right)$ (cyan line).

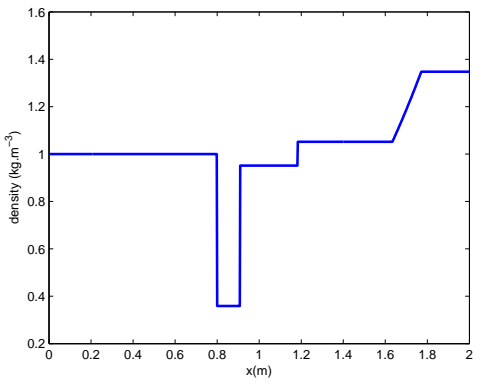

Fig. 4.23. Density for the data in Table 4.7.

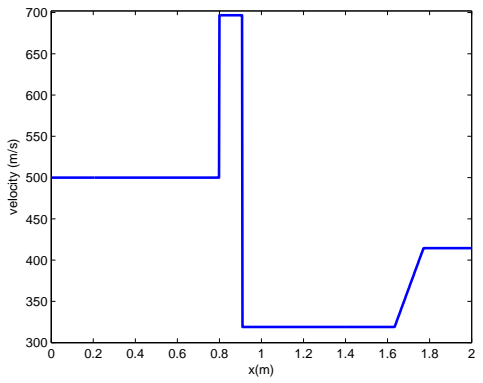

FiG. 4.24. Velocity for the data in Table 4.7.

$F$, see Figures 4.1, 4.17 and 4.18. The areas $a_{T}$ and $a_{S}$ are, respectively, defined in (3.21) and (3.22). Theorem 3.4 and Lemma 3.5 imply that in this case both states $\mathbf{J}\left(a_{R} ; \mathbf{U}_{L}, a_{L}\right)$ and $\mathbf{J}\left(a_{R} ; S_{1}^{0}\left(\mathbf{U}_{L}\right), a_{L}\right)$ exist. Therefore, the curve $C_{L}\left(\mathbf{U}_{L}\right)$ consists four parts with the same definition (4.2) as in the Case III.

Note that the segment $Q_{3}\left(\mathbf{U}_{L}\right)$ has two boundary points $\overline{\mathbf{U}}_{L}=\mathbf{J}\left(a_{R} ; \hat{\mathbf{U}}_{L}, a_{L}\right)$ and $\hat{\mathbf{U}}_{L}=S_{1}^{0}\left(\overline{\mathbf{U}}_{L}\right)$, where $\hat{\mathbf{U}}_{L}=S_{1}^{0}\left(\mathbf{U}_{L}\right)$, as well as $\overline{\mathbf{U}}_{L}=\mathbf{J}\left(a_{R} ; \mathbf{U}_{L}, a_{L}\right)$. Since $a_{L}>a_{R}$, the duct area $a$ decreases when it is varying from $a_{L}$ to $a_{R}$. According to the Corollary A.4 the corresponding velocities and pressures satisfy $\overline{\hat{v}}_{L}>\hat{\bar{v}}_{L}$ and $\overline{\hat{p}}_{L}<\hat{\bar{p}}_{L}$. This indicates that the curve $C_{L}\left(\mathbf{U}_{L}\right)$ is folding in the phase plane $(v, p)$, see Figure 4.25.

Obviously, if the intersection point of the $\mathrm{L}-\mathrm{M}$ curve $C_{L}\left(\mathbf{U}_{L}\right)$ and the $\mathrm{R}-\mathrm{M}$ curve $C_{R}\left(\mathbf{U}_{R}\right)$ lies on the segment $Q_{3}\left(\mathbf{U}_{L}\right)$, we can also find two other intermediate states, respectively, on the segments $Q_{2}\left(\mathbf{U}_{L}\right)$ and $Q_{4}\left(\mathbf{U}_{L}\right)$. That is to say, there are three solutions for one given initial data. For simplicity, denote the three intermediate 


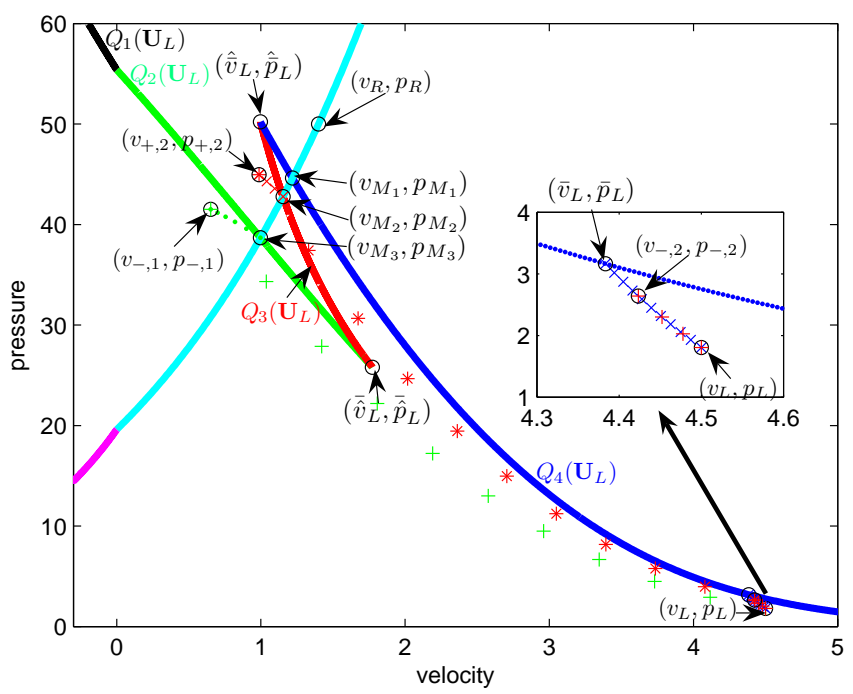

FIG. 4.25. Wave curves for the Riemann initial data in Table 4.8. The $L-M$ curve $C_{L}\left(\mathbf{U}_{L}\right)=$ $\bigcup_{k=1}^{4} Q_{k}\left(\mathbf{U}_{L}\right)$ The left wave curve $W_{L}\left(\mathbf{U}_{L}\right)$ for $\left(v_{M_{1}}, p_{M_{1}}\right) \in Q_{2}\left(\mathbf{U}_{L}\right)$ : the negative shock wave from $\left(v_{L}, p_{L}\right)$ to $\left(v_{-, 1}, p_{-, 1}\right)$ (green +$)$, then the stationary wave from $\left(v_{-, 1}, p_{-, 1}\right)$ to $\left(v_{M_{1}}, p_{M_{1}}\right)$ (green -). The left wave curve $W_{L}\left(\mathbf{U}_{L}\right)$ for $\left(v_{M_{2}}, p_{M_{2}}\right) \in Q_{3}\left(\mathbf{U}_{L}\right)$ : the stationary wave from $\left(v_{L}, p_{L}\right)$ to $\left(v_{-, 2}, p_{-, 2}\right)($ red +$)$, then the $0-$ speed shock wave from $\left(v_{-, 2}, p_{-, 2}\right)$ to $\left(v_{+, 2}, p_{+, 2}\right)$ (red $\left.*\right)$, finally the stationary wave from $\left(v_{+, 2}, p_{+, 2}\right)$ to $\left(v_{M_{2}}, p_{M_{2}}\right)(r e d \times)$. The left wave curve $W_{L}\left(\mathbf{U}_{L}\right)$ for $\left(v_{M_{3}}, p_{M_{3}}\right) \in$ $Q_{4}\left(\mathbf{U}_{L}\right)$ : the stationary wave from $\left(v_{L}, p_{L}\right)$ to $\left(\bar{v}_{L}, \bar{p}_{L}\right)$ (blue $\left.\times\right)$, then the 1-wave from $\left(\bar{v}_{L}, \bar{p}_{L}\right)$ to $\left(v_{M_{3}}, p_{M_{3}}\right)$ (blue line). The right wave curve $W_{R}\left(\mathbf{U}_{R}\right)$ is the 3 -wave from $\left(v_{M}^{k}, p_{M}^{k}\right)$ to $\left(v_{R}, p_{R}\right)$, (cyan line) $k=1,2,3$.

states as $\left(v_{M_{1}}, p_{M_{1}}\right)=Q_{2}\left(\mathbf{U}_{L}\right) \cap C_{R}\left(\mathbf{U}_{R}\right),\left(v_{M_{2}}, p_{M_{2}}\right)=Q_{3}\left(\mathbf{U}_{L}\right) \cap C_{R}\left(\mathbf{U}_{R}\right)$, and $\left(v_{M_{3}}, p_{M_{3}}\right)=Q_{4}\left(\mathbf{U}_{L}\right) \cap C_{R}\left(\mathbf{U}_{R}\right)$.

Here we use the Riemann initial data in Table 4.8 to give an example of the nonunique solutions. The $\mathrm{L}-\mathrm{M}$ curve $C_{L}\left(\mathbf{U}_{L}\right)$ is given in Figure 4.25. The exact solutions at $t=0.2$ of the density and velocity are shown in Figures 4.26 and 4.27. The letters of the possible wave configurations $A, E$ and $F$ are used to distinguish the different solutions.

TABLE 4.8

The Riemann initial data for three solutions in Case IV.

\begin{tabular}{|l|l|l|l|l|}
\hline & $a(x)$ & $\rho$ & $v$ & $p$ \\
\hline$V_{L}$ & 8.0 & 2.122 & 4.5 & 1.805 \\
\hline$V_{R}$ & 5.5 & 14.0 & 1.4 & 50.0 \\
\hline
\end{tabular}

4.1.5. Case V: $v_{L}>c_{L} ; a_{L}>a_{S}>a_{R}>a_{T}$. In this case the possible wave configurations with positive intermediate velocity are the wave configurations $A, B, E$, $F$ and $G$, see Figures 4.1, 4.2, 4.17, 4.18 and 4.31. It is necessary to introduce the wave configuration $G$, which consists of the resonant wave $S 0 S R_{1}\left(a_{L}, \mathbf{U}_{L}, a_{R}\right)$, a contact discontinuity and a 3 -wave. The resonant wave $S 0 S R_{1}\left(a_{L}, \mathbf{U}_{L}, a_{R}\right)$ is constituted of two parts, the first part is the resonant wave $S 0 S_{1}\left(a_{L}, \mathbf{U}_{L}, a_{R}\right)$ but with the sonic 


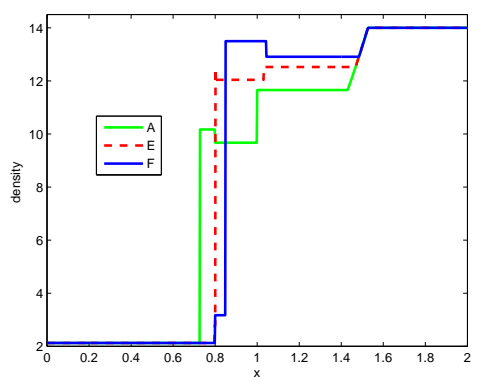

FIG. 4.26. Densities for the data in Table 4.8.

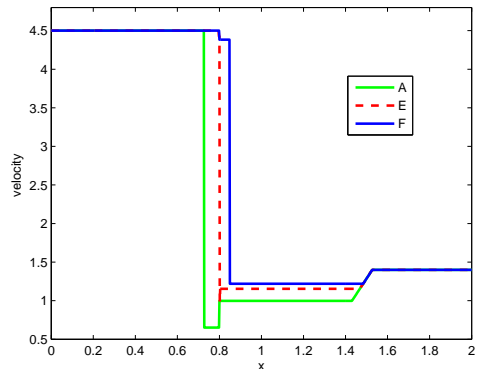

FIG. 4.27. Velocities for the data in Table 4.8.

outflow state, the second part is the transonic rarefaction wave along $T_{1}\left(\mathbf{U}_{c}^{*}\right)$ fanning from $\left(v_{c}^{*}, p_{c}^{*}\right)$ to $\left(v_{M}, p_{M}\right)$. We will explain the sonic state $\mathbf{U}_{c}^{*}$ later.

The fundamental reason for the present of the resonant wave $S 0 S R_{1}\left(a_{L}, \mathbf{U}_{L}, a_{R}\right)$ is that $a_{L}>a_{S}>a_{R}>a_{T}$. According to Theorem 3.4 and Lemma 3.5, this condition suggests that the state $\mathbf{J}\left(a_{R} ; \mathbf{U}_{L}, a_{L}\right)$ exits but that the state $\mathbf{J}\left(a_{R} ; S_{1}^{0}\left(\mathbf{U}_{L}\right), a_{L}\right)$ fails to exist. To be precise, the joint state $\overline{\mathbf{U}}_{L}$ of $Q_{2}\left(\mathbf{U}_{L}\right)$ and $Q_{3}\left(\mathbf{U}_{L}\right)$ defined in Case IV does not exist any more. Actually it bifurcates into two curves: $Q_{5}\left(\mathbf{U}_{L}\right)$ and $Q_{6}\left(\mathbf{U}_{L}\right)$, see Figure 4.28.

Before considering $Q_{5}\left(\mathbf{U}_{L}\right)$ and $Q_{6}\left(\mathbf{U}_{L}\right)$, we need to present the right boundary points of $Q_{2}\left(\mathbf{U}_{L}\right)$ and $Q_{3}\left(\mathbf{U}_{L}\right)$ in this case first. Specifically, we introduce the state $\mathbf{U}_{l}^{*}=T_{1}\left(\mathbf{U}_{L}\right) \cap\left\{\mathbf{U} \mid\left(\frac{v_{-}}{c_{-}}\right)^{2}=\beta_{l}\right\}$, where $\beta_{l}$ is defined in the Theorem 3.4. Then the sonic state $\mathbf{U}_{c}=\mathbf{J}\left(a_{R} ; \mathbf{U}_{l}^{*}, a_{L}\right)$ is the right boundary of $Q_{2}\left(\mathbf{U}_{L}\right)$. Analogously, the right boundary of $Q_{3}\left(\mathbf{U}_{L}\right)$ is also the sonic state $\mathbf{U}_{c}^{*}$. Here the superscript '*' is used to distinguish it from the sonic state $\mathbf{U}_{c}$. The sonic state $\mathbf{U}_{c}^{*}$ satisfies $\mathbf{U}_{c}^{*}=\mathbf{J}\left(a_{R} ; \mathbf{U}_{+}, a_{c}\right)$ where $\mathbf{U}_{+}=S_{1}^{0}\left(\mathbf{U}_{-}\right)$and $\mathbf{U}_{-}=\mathbf{J}\left(\mathbf{U}_{L}, a_{L} ; a_{c}\right)$. The area $a_{c}$ is the duct area at which the standing shock is located. Corollary A.5 presents the procedure for calculating $\mathbf{U}_{c}^{*}$ and $a_{c}$.

Naturally the subsequent parts $Q_{5}\left(\mathbf{U}_{L}\right)$ and $Q_{6}\left(\mathbf{U}_{L}\right)$ consists of, respectively, $T_{1}\left(\mathbf{U}_{c}\right)$ and $T_{1}\left(\mathbf{U}_{c}^{*}\right)$.

Consequently, the L-M wave curve is $C_{L}\left(\mathbf{U}_{L}\right)=\bigcup_{k=1}^{6} Q_{k}\left(\mathbf{U}_{L}\right)$. All the parts are defined as follows

$$
\begin{aligned}
& Q_{1}\left(\mathbf{U}_{L}\right)=\left\{\mathbf{U} \mid \mathbf{U} \in T_{1}\left(\mathbf{U}_{L}\right) \text { with } v \leq 0\right\}, \\
& Q_{2}\left(\mathbf{U}_{L}\right)=\left\{\mathbf{U} \mid \mathbf{U}=\mathbf{J}\left(a_{R} ; \mathbf{U}_{-}, a_{L}\right) \text { and } \mathbf{U}_{-} \in S_{1}^{-}\left(\mathbf{U}_{L}\right) \text { with }\left(\frac{v_{-}}{c_{-}}\right)^{2} \leq \beta_{l}, v<v_{c}\right\}, \\
& Q_{3}\left(\mathbf{U}_{L}\right)=\left\{\mathbf{U} \mid \mathbf{U}=\mathbf{J}\left(a_{R} ; \mathbf{U}_{+}, a\right) ; \mathbf{U}_{+}=S_{0}\left(\mathbf{U}_{-}\right) ; \mathbf{U}_{-}=\mathbf{J}\left(a ; \mathbf{U}_{L}, a_{L}\right), a_{R} \leq a \leq a_{c}\right\}, \\
& Q_{4}\left(\mathbf{U}_{L}\right)=\left\{\mathbf{U} \mid \mathbf{U} \in T_{1}\left(\overline{\mathbf{U}}_{L}\right) \text { with } v>\overline{\hat{v}}_{L}\right\}, \\
& Q_{5}\left(\mathbf{U}_{L}\right)=\left\{\mathbf{U} \mid \mathbf{U} \in T_{1}\left(\mathbf{U}_{c}\right) \text { with } v>v_{c}\right\}, \\
& Q_{6}\left(\mathbf{U}_{L}\right)=\left\{\mathbf{U} \mid \mathbf{U} \in T_{1}\left(\mathbf{U}_{c}^{*}\right) \text { with } v>v_{c}^{*}\right\} .
\end{aligned}
$$

Obviously, the $\mathrm{L}-\mathrm{M}$ wave curve $C_{L}\left(\mathbf{U}_{L}\right)$, see Figure 4.28 , in this case is also folding on the $(v, p)$ phase space. It consists of three branches $Q_{1}\left(\mathbf{U}_{L}\right) \cup Q_{2}\left(\mathbf{U}_{L}\right) \cup Q_{5}\left(\mathbf{U}_{L}\right)$, $Q_{3}\left(\mathbf{U}_{L}\right) \cup Q_{6}\left(\mathbf{U}_{L}\right)$ and $Q_{4}\left(\mathbf{U}_{L}\right)$. Apparently, if $\left(v_{M}, p_{M}\right)$ belongs to $Q_{3}\left(\mathbf{U}_{L}\right), Q_{4}\left(\mathbf{U}_{L}\right)$, $Q_{5}\left(\mathbf{U}_{L}\right)$ and $Q_{6}\left(\mathbf{U}_{L}\right)$, there are three possible solutions with the same initial data.

Andrianov and Warnecke in [1] validated the nonuniqueness using the Riemann solution by the Riemann initial data shown in Table 4.9. With their initial data we 
obtain $a_{T}=0.017386$ and $a_{S}=0.509167$. The condition of the Case $\mathbf{V}$ is satisfied. Figure 4.28 presents the corresponding $\mathrm{L}-\mathrm{M}$ curve $C_{L}\left(\mathbf{U}_{L}\right)$ and $\mathrm{R}-\mathrm{M}$ curve $C_{R}\left(\mathbf{U}_{R}\right)$. The density and velocity of the three solutions at $t=0.35$ are shown in Figures 4.29 and 4.30. We can see that the exact solutions are the same as theirs except for the extra one in red dashed line with the resonant wave, because they did not consider solutions with a resonant wave.

Besides, if $\left(v_{M}, p_{M}\right) \in Q_{5}\left(\mathbf{U}_{L}\right)$, the wave configuration is shown in Figures 4.2. But we should keep in mind that now the first 1 -wave $W$ of the resonant wave $W S W_{1}\left(\mathbf{U}_{L}, a_{L}, a_{R}\right)$ is a negative shock. If the intermediate state $\left(v_{M}, p_{M}\right) \in Q_{6}\left(\mathbf{U}_{L}\right)$, the wave configuration is shown in Figures 4.31. We exemplify these wave configurations by the Riemann initial data in Table 4.10. The corresponding wave curves are shown in Figure 4.33. Since an example with $\left(v_{M}, p_{M}\right) \in Q_{4}\left(\mathbf{U}_{L}\right)$ has been given in Case IV, we here just present the L-M wave curves $W_{L}\left(\mathbf{U}_{L}\right)$ in terms of $\left(v_{M}, p_{M}\right)$ belonging to $Q_{5}\left(\mathbf{U}_{L}\right)$ and $Q_{6}\left(\mathbf{U}_{L}\right)$. The corresponding density and velocity at $t=0.35$ are shown in Figures 4.34 and 4.35 .

TABLE 4.9

The Riemann initial data for three solutions in Case V by Andrianov and Warnecke [1] .

\begin{tabular}{|l|l|l|l|l|}
\hline & $a(x)$ & $\rho$ & $v$ & $p$ \\
\hline$V_{L}$ & 0.8 & 0.2069 & 3.991 & 0.07 \\
\hline$V_{R}$ & 0.3 & 0.1345 & -3.1668 & 0.0833 \\
\hline
\end{tabular}

TABLE 4.10

The Riemann initial data for three solutions in terms of the wave configurations $B, G$ and $F$ in Case V.

\begin{tabular}{|l|l|l|l|l|}
\hline & $a(x)$ & $\rho$ & $v$ & $p$ \\
\hline$V_{L}$ & 0.8 & 0.2069 & 3.991 & 0.07 \\
\hline$V_{R}$ & 0.3 & 0.1345 & 4.03454 & 2.7788 \\
\hline
\end{tabular}

4.1.6. Case VI: $v_{L}>c_{L} ; a_{L}>a_{S}>a_{T}>a_{R}$. In this case the possible wave configurations with positive intermediate velocity are the wave configurations $A$ and $B$, refer to Figures 4.1 and 4.2 respectively.

Due to $a_{L}>a_{S}>a_{T}>a_{R}$ according to Theorem 3.4 and Lemma 3.5, both points $\mathbf{J}\left(a_{R} ; \mathbf{U}_{L}, a_{L}\right)$ and $\mathbf{J}\left(a_{R} ; S_{1}^{0}\left(\mathbf{U}_{L}\right), a_{L}\right)$ fail to exist. Therefore, the curve $C_{L}\left(\mathbf{U}_{L}\right)$ consists only of three segments

$$
\begin{aligned}
& Q_{1}\left(\mathbf{U}_{L}\right)=\left\{\mathbf{U} \mid \mathbf{U} \in T_{1}\left(\mathbf{U}_{L}\right) \text { with } v \leq 0\right\} \\
& Q_{2}\left(\mathbf{U}_{L}\right)=\left\{\mathbf{U} \mid \mathbf{U}=\mathbf{J}\left(a_{R} ; \mathbf{U}_{-}, a_{L}\right) \text { and } \mathbf{U}_{-} \in S_{1}^{-}\left(\mathbf{U}_{L}\right) \text { with }\left(\frac{v_{-}}{c_{-}}\right)^{2} \leq \beta_{l}, v<v_{c}\right\} \\
& Q_{3}\left(\mathbf{U}_{L}\right)=\left\{\mathbf{U} \mid \mathbf{U} \in T_{1}\left(\mathbf{U}_{c}\right) \text { with } v>v_{c}\right\}
\end{aligned}
$$

We observe that the curve $C_{L}\left(\mathbf{U}_{L}\right)$ in this case is just one branch of Case $\mathbf{V}$. It is a continuous and decreasing curve in the $(v, p)$ plane. The solution is unique in this case.

4.2. The algorithm for exact Riemann solutions. In this section we present a procedure for solving exactly the Riemann problem of compressible Euler equations in a duct with discontinuous diameters. For any given Riemann initial data the L$\mathrm{M}$ and $\mathrm{R}-\mathrm{M}$ curves can be constructed in accordance with Section 4.1. As we have mentioned, the algorithm for the exact Riemann solutions has two steps, the first, 


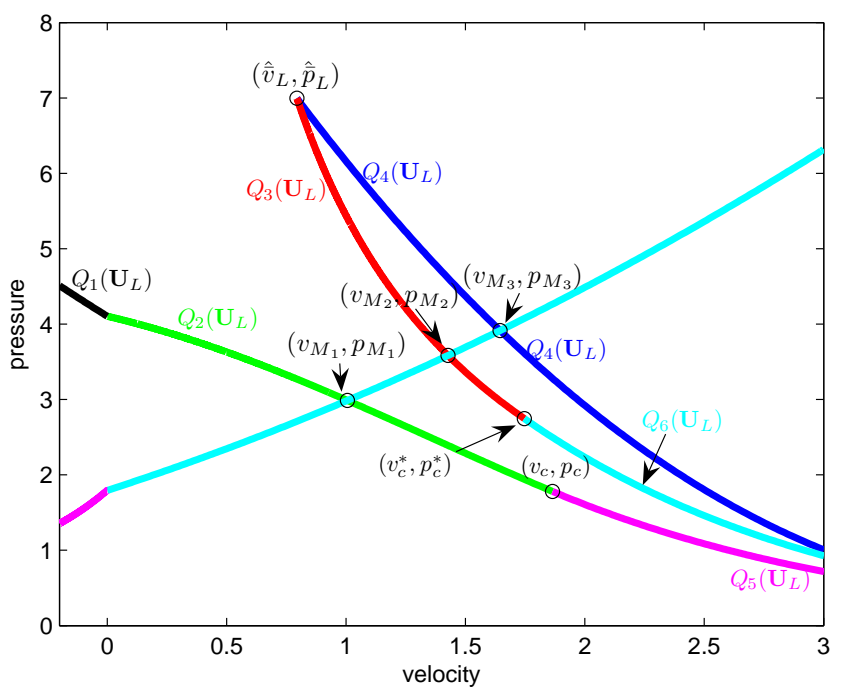

FIG. 4.28. The $L-M$ curve $C_{L}\left(\mathbf{U}_{L}\right)=\bigcup_{k=1}^{6} Q_{k}\left(\mathbf{U}_{L}\right)$ for the Riemann initial data in Table 4.9.

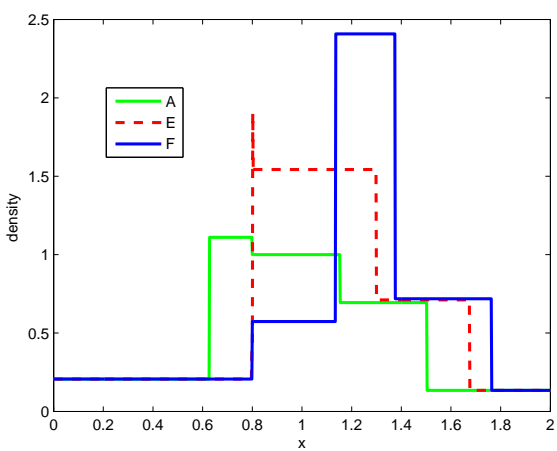

Fig. 4.29. Densities for the data in Table 4.9.

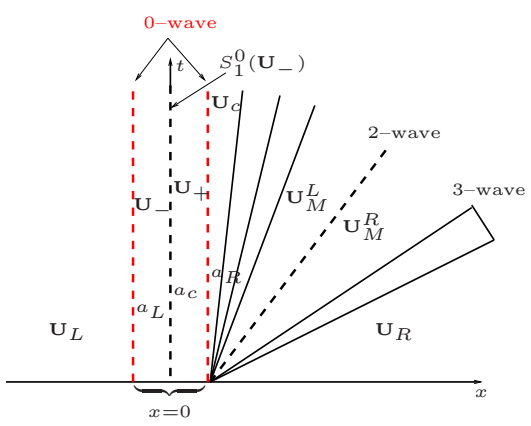

FIG. 4.31. Wave configuration $G$

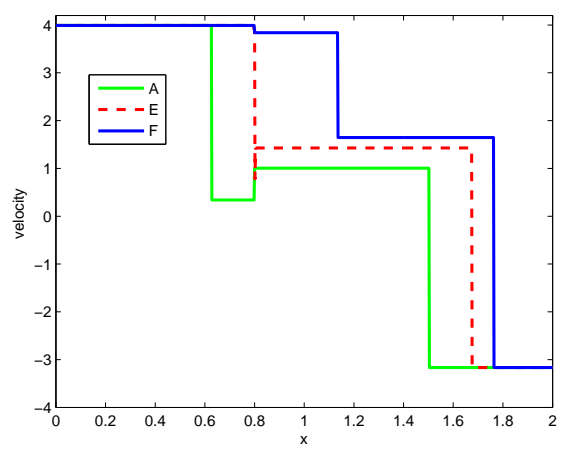

FIG. 4.30. Velocities for the data in Table 4.9.

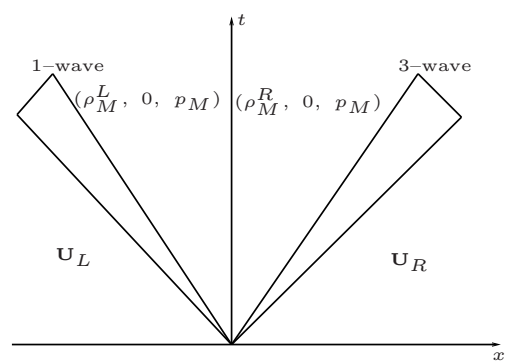

FIG. 4.32. Wave configuration $H$ 


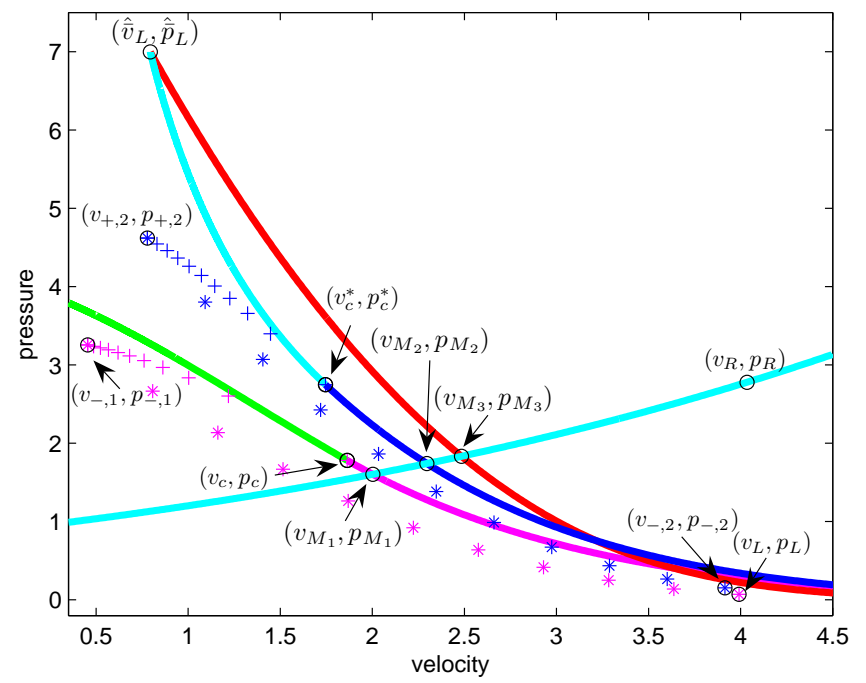

FIG. 4.33. Wave curves for the Riemann initial data in Table 4.10. The left wave curve $W_{L}\left(\mathbf{U}_{L}\right)$ for $\left(v_{M_{1}}, p_{M_{1}}\right) \in Q_{5}\left(\mathbf{U}_{L}\right)$ : the negative shock wave from $\left(v_{L}, p_{L}\right)$ to $\left(v_{-, 1}, p_{-, 1}\right)$ (magenta $\left.*\right)$, then the stationary wave from $\left(v_{-, 1}, p_{-, 1}\right)$ to $\left(v_{c}, p_{c}\right)$ (magenta +$)$, finally the 1 -wave from $\left(v_{c}, p_{c}\right)$ to $\left(v_{M_{1}}, p_{M_{1}}\right)$ (magenta line). The left wave curve $W_{L}\left(\mathbf{U}_{L}\right)$ for $\left(v_{M_{2}}, p_{M_{2}}\right) \in Q_{6}\left(\mathbf{U}_{L}\right)$ : the stationary wave from $\left(v_{L}, p_{L}\right)$ to $\left(v_{-, 2}, p_{-, 2}\right)$, then the 0-speed shock wave from $\left(v_{-, 2}, p_{-, 2}\right)$ to $\left(v_{+, 2}, p_{+, 2}\right)$ (blue $\left.*\right)$, next the stationary wave from $\left(v_{+, 2}, p_{+, 2}\right)$ to $\left(v_{c}^{*}, p_{c}^{*}\right)$, (blue +$)$, finally the 1 -wave from $\left(v_{c}^{*}, p_{c}^{*}\right)$ to $\left(v_{M_{2}}, p_{M_{2}}\right)$ (blue line). The right wave curve $W_{R}\left(\mathbf{U}_{R}\right)$ is the 3 -wave from $\left(v_{R}, p_{R}\right)$ to $\left(v_{M}^{k}, p_{M}^{k}\right)$ (cyan line) $k=$ $1,2,3$.
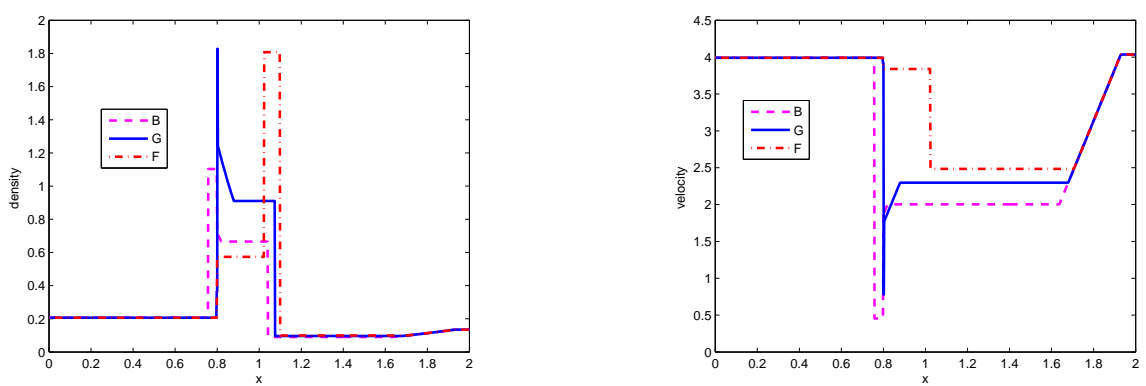

FIG. 4.34. Densities for the data in Table 4.10. FIG. 4.35. Velocities for the data in Table 4.10.

which is the most difficult one, is to calculate $\left(v_{M}, p_{M}\right)$ from the given Riemann initial data $\mathbf{V}_{L}$ and $\mathbf{V}_{R}$ by finding the intersection point of the corresponding $\mathrm{L}-\mathrm{M}$ and $\mathrm{R}-\mathrm{M}$ curves. The second step is to connect the obtained intermediate states $\left(v_{M}, p_{M}\right)$ with the initial Riemann states $\mathbf{V}_{L}$ and $\mathbf{V}_{R}$ by admissible waves to complete the solutions. In the absence of the bifurcation the $\mathrm{L}-\mathrm{M}$ curve is decreasing and the $\mathrm{R}-\mathrm{M}$ curve is increasing in the $(v, p)$ phase space. This monotonicity behavior of the curves, see the Appendix A, guarantees that the intersection point exists and is unique. Note that we assume to consider the Riemann data that do not involve a vacuum state in the solution. On the other hand, in the presence of the bifurcation, the $\mathrm{L}-\mathrm{M}$ curve consists of more than one branches. However, every branches of the L-M curve are, 
respectively, continuous and monotonic, see the Appendix A. Therefore, we can say that every solution exists and is unique on the corresponding branch.

We know that the sign of the intermediate velocity $v_{M}$ determines the position of the stationary wave on the $\mathrm{L}-\mathrm{M}$ curve or $\mathrm{R}-\mathrm{M}$ curve. To verify the sign of the intermediate velocity, we calculate two pressures $p_{l}^{0}$ and $p_{r}^{0}$ which satisfy

$$
p_{l}^{0}=T_{1}\left(\mathbf{U}_{L}\right) \cap\{v \mid v=0\}, \quad p_{r}^{0}=T_{2}\left(\mathbf{U}_{R}\right) \cap\{v \mid v=0\} .
$$

Due to the restriction (2.15) at least one of the pressures in (4.3) is positive. Therefore if $p_{l}^{0}>p_{r}^{0}$, the intermediate velocity $v_{M}>0$; or else if $p_{r}^{0}>p_{l}^{0}, v_{M}<0$. One trivial case is when $p_{l}^{0}=p_{r}^{0}$, i.e. $v_{M}=0$. In such a case both the stationary wave and the contact discontinuity disappear and the trivial resonant wave corresponding to $\lambda_{2}=\lambda_{0}$ occurs. The wave configuration of the exact solution is shown in Figure 4.32.

Now we demonstrate the algorithm by assuming that $a_{L}>a_{R}>a_{S}>a_{T}$ and $v_{L}-c_{L}>0$ as well as $v_{R}+c_{R} \geq 0$, i.e. the $\mathrm{L}-\mathrm{M}$ curve $C_{L}\left(\mathbf{U}_{L}\right)$ is defined in (4.2) belonging to Case IV; the R-M curve $C_{R}\left(\mathbf{U}_{R}\right)$ is in Case II. To simplify the process, we present the procedure for the case $p_{l}^{0}>p_{r}^{0}$. Due to the fact that the $\mathrm{L}-\mathrm{M}$ curve contains the bifurcation in Case $\mathbf{I V}$, there may be more than one solutions, see Figure 4.25. Before presenting the details of the exact Riemann solution algorithm, we define

$$
f_{q}\left(p ; \rho_{q}, p_{q}\right)=\left\{\begin{array}{cc}
\left(p-p_{q}\right) \sqrt{\frac{\left(1-\mu^{2}\right) / \rho_{q}}{p_{q}+\mu^{2} p}} & p>p_{q} \\
\frac{2 c_{q}}{\gamma-1}\left[\left(\frac{p}{p_{q}}\right)^{\frac{\gamma-1}{2 \gamma}}-1\right] & p \leq p_{q}
\end{array}\right.
$$

where $q$ is $L$ or $R$. The algorithm is as follows.

Step 1. Calculate $p_{l}^{0}$ and $p_{r}^{0}$ from (4.3).

$$
\text { If } p_{l}^{0}>p_{r}^{0}, a_{L}>a_{R}>a_{S}>a_{T} \text { and } v_{L}-c_{L}>0 \text {, go to Step } 2 \text {. }
$$

Step 2. Compute two boundary states $\overline{\mathbf{U}}_{L}=\mathbf{J}\left(S_{1}^{0}\left(\mathbf{U}_{L}\right), a_{L} ; a_{R}\right)$ and $\hat{\mathbf{U}}_{L}=S_{1}^{0}\left(\mathbf{J}\left(\mathbf{U}_{L}, a_{L} ; a_{R}\right)\right)$, then calculate two velocities $v_{1}=v_{R}+f_{R}\left(\hat{\bar{p}}_{L} ; \rho_{R}, p_{R}\right)$ and $\left.v_{2}=v_{R}+f_{R}\left(\overline{\hat{p}}_{L} ; \rho_{R}, p_{R}\right)\right)$.

Step 3. a) If $v_{1} \leq \hat{\bar{v}}_{L}$, there is exactly the one solution shown in Figure 4.1. Solve the system (4.5) to obtain it.

b) If $v_{1}>\hat{\bar{v}}_{L}$ and $v_{2}<\overline{\hat{v}}_{L}$, there are three possible solutions.

1. Solve the system (4.5) to obtain the first possible solution shown in Figure 4.1.

2. Solve the system (4.7) to obtain the second possible solution shown in Figure 4.17.

3. Solve the system (4.6) to obtain the third possible solution shown in Figure 4.18.

c) If $v_{2} \geq \overline{\hat{v}}_{L}$, there is exactly the one solution shown in Figure 4.18. Solve the system (4.6) to obtain it.

The systems (4.5), (4.6) and (4.7) are defined in the following 


$$
\left\{\begin{array}{l}
v_{-}=v_{L}-f_{L}\left(p_{-} ; \rho_{L}, p_{L}\right) \\
\rho_{-}=g_{L}\left(p_{-} ; \rho_{L}, p_{L}\right) \\
\mathbf{U}_{M}^{L}=\mathbf{J}\left(a_{R} ; \mathbf{U}_{-}, a_{L}\right) \\
v_{M}=v_{R}+f_{R}\left(p_{M} ; \rho_{R}, p_{R}\right) \\
\rho_{M}^{R}=g_{R}\left(p_{M} ; \rho_{R}, p_{R}\right)
\end{array}\right.
$$

$(4.6) \quad\left\{\begin{array}{l}\overline{\mathbf{U}}_{L}=\mathbf{J}\left(a_{R} ; \mathbf{U}_{L}, a_{L}\right), \\ v_{M}=\bar{v}_{L}-f_{L}\left(p_{M} ; \bar{\rho}_{L}, \bar{p}_{L}\right), \\ \rho_{M}^{L}=g_{L}\left(p_{M} ; \bar{\rho}_{L}, \bar{p}_{L}\right), \\ v_{M}=v_{R}+f_{R}\left(p_{M} ; \rho_{R}, p_{R}\right), \\ \rho_{M}^{R}=g_{R}\left(p_{M} ; \rho_{R}, p_{R}\right) .\end{array}\right.$

$$
(4.7)\left\{\begin{array}{l}
\mathbf{U}_{-}=\mathbf{J}\left(a_{R} ; \mathbf{U}_{L}, a_{L}\right), \\
p_{+}=p_{-}\left[\left(1+\mu^{2}\right)\left(\frac{v_{-}}{c_{-}}\right)^{2}-\mu^{2}\right], \\
\rho_{+}=\rho_{-} \frac{\left(\frac{v_{-}}{c_{-}}\right)^{2}}{\mu^{2}\left(\frac{v_{-}}{c_{-}}\right)^{2}+\left(1-\mu^{2}\right)} \\
v_{+}=v_{-}-\left(1-\mu^{2}\right) \frac{c_{-}^{2}}{v_{-}}\left[\left(\frac{v_{-}}{c_{-}}\right)^{2}-1\right], \\
\mathbf{U}_{M}^{L}=\mathbf{J}\left(a_{R} ; \mathbf{U}_{+}, a_{L}\right) \\
v_{M}^{R}=v_{R}+f_{R}\left(p_{M} ; \rho_{R}, p_{R}\right), \\
\rho_{M}^{R}=g_{R}\left(p_{M} ; \rho_{R}, p_{R}\right) .
\end{array}\right.
$$

We emphasize that all these nonlinear systems (4.5), (4.6), and (4.7) have a unique solution and can be transformed into a series of simple and monotonic algebra systems. Therefore iteration methods, e.g. the Newton-Raphson method, can be applied to obtain the solution.

4.3. Conclusions. For any given Riemann initial data $\mathbf{V}_{L}$ and $\mathbf{V}_{R}$, we obtained all possible exact solutions to the Euler equations in a duct with discontinuous crosssection by constructing the $\mathrm{L}-\mathrm{M}$ and $\mathrm{R}-\mathrm{M}$ curves. For each curve, there are six different cases in accordance with variations of the duct areas and the initial Mach number. We analyze the behavior of the $\mathrm{L}-\mathrm{M}$ and $\mathrm{R}-\mathrm{M}$ curves in each case. We observe that if the given initial data belong to Cases IV and $\mathbf{V}$, there may be more than one possible solution due to a bifurcation on the $\mathrm{L}-\mathrm{M}$ or $\mathrm{R}-\mathrm{M}$ curves.

According to Liu [12] there may be three solutions along a contracting duct for the same given boundary values. The one with a standing shock wave is unstable. That is to say the solution with the wave configuration $E$ in Cases $\mathbf{I V}$ and $\mathbf{V}$ is unphysical. However there are still at least two solutions left. One needs to consider an additional criterion to select the physically relevant solution. For the classical solutions, Andrianov and Warnecke in [1] chose one by a kind of entropy rate admissibility condition, see Dafermos [5], out of all possible solutions. Their consideration was based on the comparision of the solutions of the governing equations with $2 \mathrm{D}$ computations of the usual Euler equations in a tube with corresponding geometry, i.e. a jump in the cross section. We believe that the nonuniqueness problem is related to the application of the model. In the future we intend to compare all nonunique solutions with the numerical results of the Euler equations in an axisymmetric tube. This was suggested by Rochette et al. [16] to find a criterion for physically relevant solutions and seems even more appropriate.

Appendix A. Monotonic behavior of wave curves.

Lemma A.1. The curve $Q\left(\mathbf{U}_{L}\right)$ defined in (A.1) is monotone decreasing in the $(v, p)$ phase plane.

$(\mathrm{A} .1) Q\left(\mathbf{U}_{L}\right)=\left\{\mathbf{U} \mid \mathbf{U}=\mathbf{J}\left(a_{R} ; \mathbf{U}_{-}, a_{L}\right)\right.$ with $\mathbf{U}_{-} \in T_{1}\left(\mathbf{U}_{L}\right)$ and $\left.0<\frac{v_{-}}{c_{-}}<\alpha\right\}$,

where

$$
\alpha= \begin{cases}1, & a_{L}<a_{R}, \\ \sqrt{\beta_{l}}, & a_{L}>a_{R},\end{cases}
$$

and $\beta_{l}$ is defined in Theorem 3.4. 
Proof. Given two states $\mathbf{U}_{k} \in Q\left(\mathbf{U}_{L}\right), k=1,2$, our aim is to prove that if $0<v_{1}<v_{2}$ then $p_{1}>p_{2}$ Assume that $\mathbf{U}_{k,-} \in T_{1}\left(\mathbf{U}_{L}\right)$ and $\mathbf{U}_{k}=J\left(a_{R} ; \mathbf{U}_{k,-}, a_{L}\right)$. Note that the curve $T_{1}\left(\mathbf{U}_{L}\right)$ in the $(v, p)$ phase plane is decreasing. Therefore, if $0<v_{1,-}<v_{2,-}$, then $p_{1,-}>p_{2,-}$.

From the velocity functions $\Psi\left(v ; \mathbf{V}_{-}, a_{R}\right)$ defined in (3.11), we have the following relations

$$
\frac{\partial \Psi\left(v ; \mathbf{V}_{-}, a_{R}\right)}{\partial p_{-}} \begin{cases}=\frac{1}{\rho_{L}}\left(\frac{p_{-}}{p_{L}}\right)^{-\frac{1}{\gamma}}\left[v_{-}+c_{-}\left(\frac{a_{L} v_{-}}{a_{R} v}\right)^{\gamma-1}\right] \frac{v_{-}-c_{-}}{v_{-} c_{-}}, & \text {if } p_{-} \leq p_{L}, \\ <\delta\left[\frac{p_{L}^{2}+p_{-}^{2}+2 \mu^{2} p_{-} p_{L}}{c_{-}}+\kappa\left(\frac{a_{L} v_{-}}{a_{R} v}\right)^{\gamma-1}\right]\left(v_{-}-c_{-}\right), & \text {if } p_{-}>p_{L},\end{cases}
$$

where $\kappa=\frac{\left(2 \mu^{2}+1\right) p_{L}^{2}+p_{L} p_{-}}{v_{-}}$and $\delta=\frac{\gamma}{(\gamma+1) \rho_{L}} \frac{1}{\left(\mu^{2} p_{L}+p_{-}\right)^{2}}$.

Thus for any subsonic state $v$, if $p_{1,-}>p_{2,-}$ we have $\Psi\left(v ; \mathbf{V}_{1,-}, a_{R}\right)<\Psi\left(v ; \mathbf{V}_{2,-}, a_{R}\right)$. Hence we have $0<v_{1}<v_{2}$. In addition from (3.4), $c_{1}>c_{2}$ holds. Due to the fact that the entropy is constant across the stationary wave, it follows that $p_{1}>p_{2}$.

Lemma A.2. Assume that $\mathbf{U}_{-} \in T_{1}\left(\mathbf{U}_{L}\right)$ and $\mathbf{U}=\mathbf{J}\left(a_{R} ; \mathbf{U}_{-}, a_{L}\right)$. The quantities $M^{2}=\left(\frac{v}{c}\right)^{2}$ and $M_{-}^{2}=\left(\frac{v_{-}}{c_{-}}\right)^{2}$ are the squared Mach number of the states $\mathbf{U}$ and $\mathbf{U}_{-}$ respectively. If $M_{-}^{2}<1$ is increased then $M^{2}<1$ also increases and vice versa.

Proof. From (3.6) and (3.9) we have

$$
a_{L} c_{-}^{\frac{2}{\gamma-1}} v_{-}=a_{R} c^{\frac{2}{\gamma-1}} v
$$

Due to the relation (3.8) we have the following relations

$$
\frac{a_{L}^{2} c_{-}^{\frac{4}{\gamma-1}} v_{-}^{2}}{\left[\frac{v_{-}^{2}}{2}+\frac{c_{-}^{2}}{\gamma-1}\right]^{\frac{\gamma+1}{\gamma-1}}}=\frac{a_{R}^{2} c^{\frac{4}{\gamma-1}} v^{2}}{\left[\frac{v^{2}}{2}+\frac{c^{2}}{\gamma-1}\right]^{\frac{\gamma+1}{\gamma-1}}}
$$

With a simple calculation, the following relation holds

$$
\frac{M^{2}}{\left(1+\frac{\gamma-1}{2} M^{2}\right)^{\frac{\gamma+1}{\gamma-1}}}=\left(\frac{a_{L}}{a_{R}}\right)^{2} \frac{M_{-}^{2}}{\left(1+\frac{\gamma-1}{2} M_{-}^{2}\right)^{\frac{\gamma+1}{\gamma-1}}} .
$$

Hence the relation (A.4) implies that the squared Mach number $M^{2}$ can be treated as a function of $M_{-}^{2}$.

We introduce the function $\phi(x)$ from (A.4)

$$
\phi(x):=\frac{x}{\left(1+\frac{\gamma-1}{2} x\right)^{\frac{\gamma+1}{\gamma-1}}} .
$$

Then (A.4) becomes $\phi\left(M^{2}\right)=\left(\frac{a_{L}}{a_{R}}\right)^{2} \phi\left(M_{-}^{2}\right)$. The derivative of the function $\phi(x)$ is

$$
\phi^{\prime}(x)=\left(1+\frac{\gamma-1}{2} x\right)^{\frac{-2 \gamma}{\gamma-1}}(1-x) \text {. }
$$

So $\phi(x)$ increases when $0<x<1$ and decreases when $x>1$. Consequently, if $M_{-}^{2}<1$ is increased then $M^{2}<1$ also increases and vice versa. $\square$

Proposition A.3. From (3.4) the velocity and the sound speed vary in the opposite direction. Hence for the positive velocity, if the Mach number $M=\frac{v}{c}$ is 
increased then $v$ increases and $c$ decreases. It follows that $p$ also decreases due to the fact that the entropy is constant. Similarly, if $M$ is decreased then $v$ decreases and $p$ increases.

Lemma A.4. Consider the curve

(A.7) $\left\{\mathbf{U} \mid \mathbf{U}=\mathbf{J}\left(a_{R} ; \mathbf{U}_{+}, a\right) ; \mathbf{U}_{+}=S_{0}\left(\mathbf{U}_{-}\right) ; \mathbf{U}_{-}=\mathbf{J}\left(a ; \mathbf{U}_{L}, a_{L}\right), a \in\right] a_{L} a_{R}[\}$.

We have

1. if the duct is expanding, the velocity increases and the pressure decreases when a varies from $a_{L}$ to $a_{R}$;

2. if the duct is contracting, the velocity decreases and the pressure increases when a varies from $a_{L}$ to $a_{R}$.

Proof. From (3.27) and (A.4), we have the relations

$$
\begin{aligned}
\frac{M_{-}^{2}}{\left(1+\frac{\gamma-1}{2} M_{-}^{2}\right)^{\frac{\gamma+1}{\gamma-1}}} & =\left(\frac{a_{L}}{a}\right)^{2} \frac{M_{L}^{2}}{\left(1+\frac{\gamma-1}{2} M_{L}^{2}\right)^{\frac{\gamma+1}{\gamma-1}}}, \\
M_{+}^{2} & =\frac{\mu^{2} M_{-}^{2}+\left(1-\mu^{2}\right)}{\left(1+\mu^{2}\right) M_{-}^{2}-\mu^{2}} \\
\frac{M^{2}}{\left(1+\frac{\gamma-1}{2} M^{2}\right)^{\frac{\gamma+1}{\gamma-1}}} & =\left(\frac{a}{a_{R}}\right)^{2} \frac{M_{+}^{2}}{\left(1+\frac{\gamma-1}{2} M_{+}^{2}\right)^{\frac{\gamma+1}{\gamma-1}}} .
\end{aligned}
$$

If $M_{L}^{2} \geq 1$, then we obtain $M_{-}^{2} \geq 1$ and $M^{2}<1$. According to the behavior of (A.5), the Mach number $M_{-}^{2}$ increases when $a$ increases and vice versa. We multiply the two equations (A.8) and (A.10) to find the relationship between the duct area $a$ and the square of Mach number $M^{2}$.

$$
\left(\frac{a_{R}}{a_{L}}\right)^{2} \frac{\left(1+\frac{\gamma-1}{2} M_{L}^{2}\right)^{\frac{\gamma+1}{\gamma-1}}}{M_{L}^{2}} \frac{M^{2}}{\left(1+\frac{\gamma-1}{2} M^{2}\right)^{\frac{\gamma+1}{\gamma-1}}}=\frac{M_{+}^{2}}{M_{-}^{2}}\left(\frac{1+\frac{\gamma-1}{2} M_{-}^{2}}{1+\frac{\gamma-1}{2} M_{+}^{2}}\right)^{\frac{\gamma+1}{\gamma-1}} .
$$

We set $m=M_{-}^{2}$. For the right part of (A.11), we introduce the function

$$
\omega(m):=\frac{s(m)}{m}\left(\frac{1+\frac{\gamma-1}{2} m}{1+\frac{\gamma-1}{2} s(m)}\right)^{\frac{\gamma+1}{\gamma-1}},
$$

where $s(m)$ was defined in (3.27).

The derivative of the function $\omega(m)$ is

$\left(\right.$ A.13) $\omega^{\prime}(m)=\frac{\left[1+\frac{\gamma-1}{2} m\right]^{\frac{2}{\gamma-1}}}{m^{2}\left[1+\frac{\gamma-1}{2} s(m)\right]^{\frac{2 \gamma}{\gamma-1}}} \quad\left\{m s^{\prime}(m)\left[1+\frac{\gamma-1}{2} m\right]\left(1+\frac{\gamma-1}{2} s(m)\right.\right.$

$$
\left.\left.-\frac{\gamma+1}{2} m s(m)\right)+(m-1) s(m)\left[1+\frac{\gamma-1}{2} s(m)\right]\right\},
$$

where

$$
s^{\prime}(m)=-\frac{1}{\left(\left(1+\mu^{2}\right) m-\mu^{2}\right)^{2}} .
$$

Since $m>1$, we obtain $s(m)>0$. Also due to $s^{\prime}(m)<0$ and the part

$$
1+\frac{\gamma-1}{2} s(m)-\frac{\gamma+1}{2} m s(m)=\frac{\left(\gamma^{2}-1\right) m(1-m)}{2(2 \gamma m-(\gamma-1))}<0 .
$$


we get $\omega^{\prime}(m)>0$, i.e. the right part of (A.11) is increasing as $m$ increases and vice versa. From (A.8), this implies that the left part of (A.11) increases and vice versa. Since the duct area is fixed and $M_{L}$ is constant, we can figure out that the part

$$
\frac{M^{2}}{\left(1+\frac{\gamma-1}{2} M^{2}\right)^{\frac{\gamma+1}{\gamma-1}}}
$$

increases when $a$ increases and vice versa. Because of $M^{2}<1$, according to the behavior of the function (A.5), we achieve that $M^{2}$ increases when $a$ increases and vice versa. This concludes the proof in accordance with the Proposition A.3.

Corollary A.5. Denote $a_{c}$ as the area which satisfies $\mathbf{U}_{c}=\mathbf{J}\left(a_{R} ; \mathbf{U}_{+}, a_{c}\right)$ where $\mathbf{U}_{+}=S_{1}^{0}\left(\mathbf{U}_{-}\right)$and $\mathbf{U}_{-}=\mathbf{J}\left(\mathbf{U}_{L}, a_{R} ; a_{c}\right)$. From relations (A.8),(A.9) and (A.10), we have

$$
\Phi(m):=\left(\frac{a_{R}}{a_{L}}\right)^{2} \frac{2}{(\gamma+1)^{\frac{\gamma+1}{\gamma-1}}} \frac{\left(1+\frac{\gamma-1}{2} M_{L}^{2}\right)^{\frac{\gamma+1}{\gamma-1}}}{M_{L}^{2}}-\omega(m)=0
$$

where $\omega(m)$ is defined in (A.12). Since the Mach number $M_{L}$ is constant and $\omega(m)$ is a monotonic function, the solution of the equation $\Phi(m)=0$ can be obtained by an iteration method, additionally $\left.m \in] 1, M_{L}^{2}\right]$. Finally after we obtain the squared Mach number $m$, the area $a_{c}$ then can be simply calculated from (A.8).

\section{REFERENCES}

[1] N. Andrianov And G. WARnecke, On the solution to Riemann problem for compressible duct flow. SIAM J. Appl. Math., 64(2004), pp. 878-901.

[2] N. Andrianov and G. Warnecke, The Riemann problem for Baer-Nunziato model of two phase flows. J. Comput. Phys., 195(2004), pp. 434-464.

[3] S. Clain And D. Rochette, First- and second-order finite volume method for the onedimensional nonconservative Euler system. J. Comput. Phys., 22(2009), pp. 8214-8248.

[4] A. Courant and K. O. Friedrichs, Supersonic Flow and Shock Waves. Springer, 1999.

[5] C. M. Dafermos, The entropy rate admissible criterion for solutions of hyperbolic conservation laws, J. Diff. Eqs., 14(1973), pp. 202-212.

[6] V. G. Dulov, Decay of an arbitrary initial discontinuity of gas parameters across a jump discontinuity in the cross-section area. Vestnik leningrad. Univ. 19(1958), pp. 76-99. (in Russian)

[7] L. C. Evans, Partial Differential Equations, Amer. Math. Soc., 1998.

[8] P. Garcia-Navarro And M. E. Vazquez-Cendom, On numercial treatment of the source terms in shallow water equations. Computer \& Fluids, 29(2000), pp. 951-979.

[9] P. Goatin And P. G. Lefloch, The Riemann problem for a class of resonant hyperbolic systems of balance laws. Ann. Inst. H. Poincaré Anal. Non Linéaire, 21(2004), pp. 881-902.

[10] E. IsaAcson And B. Temple, Nonlinear resonance in systems of conservation laws. SIAM J. Appl. Math., 52(1992), pp. 1260-1278.

[11] P. G. Lefloch And M. D. Thanh, The Rieman problem for fluid flows in a nozzle with discontinuous cross-section. Commun. Maths. Sci., 1(2003), pp. 763-797.

[12] T. P. Liu, Transonic gas flow in a duct of varying area. Arch. Rat. Mech. Anal., 23(1982), $1-18$.

[13] T. P. LIU, Nonlinear resonance for quasilinear hyperbolic equation. J. Math. Phys., 28(1987), pp. 2593-2602.

[14] C. A. Lowe, Two-phase shock-tube problems and numerical methods of solution. J. Comput. Phys., 204(2005), pp. 598-632.

[15] D. Marchesin and P. J. Paes-Leme, A Riemann problem in gas dynamics with bifurcation. Comp. Maths. Appls., 12(1986), pp. 433-455.

[16] D. Rochette, S. Clain, And W. Bussière, Unsteady compressible flow in ducts with varying cross-section: Comparison between the nonconservative Euler system and axisymmetric flow model. Preprint submitted. 
[17] J. Smoller, Shock Waves and Reaction-Diffusion Equations. Springer, 1994.

[18] M. D. Thanh, The Riemann problem for a nonisentropic fluid in a nozzle with discontinuous cross-section. SIAM J. Appl. Math., 69(2009), pp. 1501-1519.

[19] E. F. Toro, Riemann Solver and Numerical Methods for Fluid Dynamics: A Practical Intraduction. Springer, 1997. 